\title{
LI-RADS: a conceptual and historical review from its beginning to its recent integration into AASLD clinical practice guidance
}

This article was published in the following Dove Press journal: Journal of Hepatocellular Carcinoma

Khaled M Elsayes, ${ }^{\prime}$ Ania Z Kielar, ${ }^{2}$ Victoria Chernyak, ${ }^{3}$ Ali Morshid,' Alessandro Furlan, ${ }^{4}$ William R Masch, ${ }^{5}$ Robert M Marks, ${ }^{6}$ Aya Kamaya, ${ }^{7}$ Richard KG Do, ${ }^{8}$ Yuko Kono, ${ }^{9}$ Kathryn J Fowler, ${ }^{9}$ An Tang, ${ }^{10}$ Mustafa R Bashir," Elizabeth M Hecht, ${ }^{12}$ Kedar Jambhekar, ${ }^{13}$ Andrej Lyshchik, ${ }^{14}$ Shuchi K Rodgers, ${ }^{14}$ Jay $\mathrm{P}$ Heiken, ${ }^{15}$ Marc Kohli, ${ }^{16}$ David T Fetzer, ${ }^{17}$ Stephanie R Wilson, ${ }^{18}$ Zahra Kassam, ${ }^{19}$ Mishal Mendiratta-Lala, ${ }^{5}$ Amit G Singal, ${ }^{17}$ Christopher S Lim, ${ }^{20}$ Irene Cruite, ${ }^{21}$ James Lee, ${ }^{22}$ Ryan Ash, ${ }^{23}$ Donald G Mitchell, ${ }^{14}$ Matthew DF Mclnnes, ${ }^{24}$ Claude B Sirlin 9

'Department of Diagnostic Radiology, The University of Texas MD Anderson Cancer Center, Houston, TX, USA 2Department of Radiology, University of Toronto, ON, Canada; ${ }^{3}$ Department of Radiology, Montefiore Medica Center, Bronx, NY, USA; ${ }^{4}$ Department of Radiology, University of Pittsburgh, Pittsburgh, PA, USA; ${ }^{5}$ Departmen of Radiology, University of Michigan, Ann Arbor, MI, USA; ${ }^{6}$ Department of Radiology, Naval Medical Center San Diego, Uniformed Services University of the Health Sciences, Bethesda, MD, USA; 'Department of Radiology, Stanford University Medical Center, Stanford, CA, USA; ${ }^{8}$ Department of Radiology, Memorial Sloan Kettering Cancer Center, New York, NY, USA; 'Department of Radiology, University of California San Diego, CA, USA; ${ }^{10}$ Department of Radiology. Centre Hospitalier de l'Université de Montréal (CHUM). Montreal, QC, Canada; "Department of Radiology, Center for Advanced Magnetic Resonance Development, and Division of Gastroenterology, Department of Medicine, Duke University Medical Center, Durham, NC, USA; '2Department oniversity Medical Center, Durham, NC, USA; ' 2 Department of Radiology, Columbia University Medical Center, New York, NY, USA; ' 3 Department of Radiology, University of Arkansas for Medical Sciences, Little Rock, AR, USA; ' Department of Radiology, Einstein Medical Center, Philadelphia, PA, USA USA; ${ }^{6}$ Dent or Radiology, Mayo Clic, Rochester, MN, USA; "Department of Radiology, University of California San Francisco, CA, USA; "Division of Digestive and Liver Diseases, UT Southwestern Medical Center, Dallas, TX, USA ${ }^{18}$ Department of Radiology, University of Calgary, Calgary, $A B$, Canada; '9 Department of Diagnostic Imaging, Schulich School of Medicine, London, ON, Canada; ${ }^{20}$ Department of Medical Imaging, Sunnybrook Health Sciences Centre, University of Toronto, ON, Canada; ${ }^{2}$ Department of Radiology, Inland Imaging, Spokane, WA, USA; ${ }^{22}$ Departmen of Radiology, University of Kentucky, Lexington, KY, USA; ${ }^{23}$ Department of Radiology, University of Kansas, Kansas City, KS, USA; ${ }^{24}$ Department of Radiology, University of Ottawa, ON, Canada

Correspondence: Khaled M Elsayes

Department of Diagnostic Radiology, The University of Texas MD Anderson Cancer Center, 1400 Pressler Street, Houston, TX 77030, USA

TX 77030, USA

$\mathrm{Tel}+\mathrm{l} 7137453025$

\begin{abstract}
The Liver Imaging Reporting and Data System (LI-RADS ${ }^{\circledR}$ ) is a comprehensive system for standardizing the terminology, technique, interpretation, reporting, and data collection of liver observations in individuals at high risk for hepatocellular carcinoma (HCC). LI-RADS is supported and endorsed by the American College of Radiology (ACR). Upon its initial release in 2011, LI-RADS applied only to liver observations identified at CT or MRI. It has since been refined and expanded over multiple updates to now also address ultrasound-based surveillance, contrast-enhanced ultrasound for HCC diagnosis, and CT/MRI for assessing treatment response after locoregional therapy. The LI-RADS 2018 version was integrated into the HCC diagnosis, staging, and management practice guidance of the American Association for the Study of Liver Diseases (AASLD). This article reviews the major LI-RADS updates since its 2011 inception and provides an overview of the currently published LI-RADS algorithms.
\end{abstract}

Keywords: LI-RADS, v2018, CT, MRI, CEUS, US, HCC, liver imaging, reporting, cirrhosis

\section{Introduction}

Hepatocellular carcinoma (HCC) is the most common primary liver malignancy and the third leading cause of cancer-related mortality worldwide. ${ }^{1,2}$ Early detection of HCC is important as it has been shown to improve overall survival, particularly when patients are able to receive potentially curative therapy such as resection or orthotopic liver transplantation. ${ }^{3}$ The diagnosis of HCC may be made noninvasively by imaging findings alone, often without the need for percutaneous biopsy, in patients who are considered to be at high risk for HCC. ${ }^{4-6}$ Consequently, radiologists must be accurate in their interpretation and reporting of liver imaging so that therapy may be rendered to patients with HCC in an appropriate and timely manner. The Liver Imaging Reporting and Data System (LI-RADS ${ }^{\circledR}$ ) was conceived to address this need. Free-text reports often contain vague wording and may vary based on the radiologist practice setting and experience level. This, in turn, may lead to confusion among clinical teams responsible for the management of patients at risk for or with HCC. LI-RADS provides a standardized lexicon, strict diagnostic criteria, an easy-to-follow diagnostic algorithm, and reporting guidelines to improve the consistency and clarity of radiologist interpretation and reporting.

One benefit of LI-RADS is improved communication between radiologists and clinicians. According to the LI-RADS diagnostic algorithm, each liver observation in a patient at high risk for HCC is assigned a category (from LR-1 to LR-5) reflecting the relative likelihood of being HCC. ${ }^{7}$ Interrater agreement for LI-RADS categorization is substantial, and structured LI-RADS reporting has been shown to improve reporting 
consistency. ${ }^{8,9}$ These studies were largely conducted among expert radiologists at high-volume centers, and further data among community radiologists are needed. LI-RADS is supported and endorsed by the American College of Radiology (ACR) and in 2018 was integrated into the American Association for the Study of Liver Diseases (AASLD) guidance statement for HCC. ${ }^{1}$

In this article, we review the expansions and updates of LI-RADS since its 2011 inception, including the LI-RADS ultrasound surveillance algorithm, the LI-RADS CT/MRI diagnostic algorithm (Figure 1), the LI-RADS contrastenhanced ultrasound (CEUS) diagnostic algorithm, and the LI-RADS CT/MRI treatment response algorithm. Major changes to the LI-RADS v2018 CT/MRI diagnostic algorithm are highlighted, and a glimpse into future directions of LI-RADS is provided.

\section{Overview of major LI-RADS updates}

LI-RADS is a dynamic system, with updates released regularly to incorporate user feedback, expanding knowledge, and

\section{CT/MRI LI-RADS ${ }^{\circledR}$ V2018 CORE}

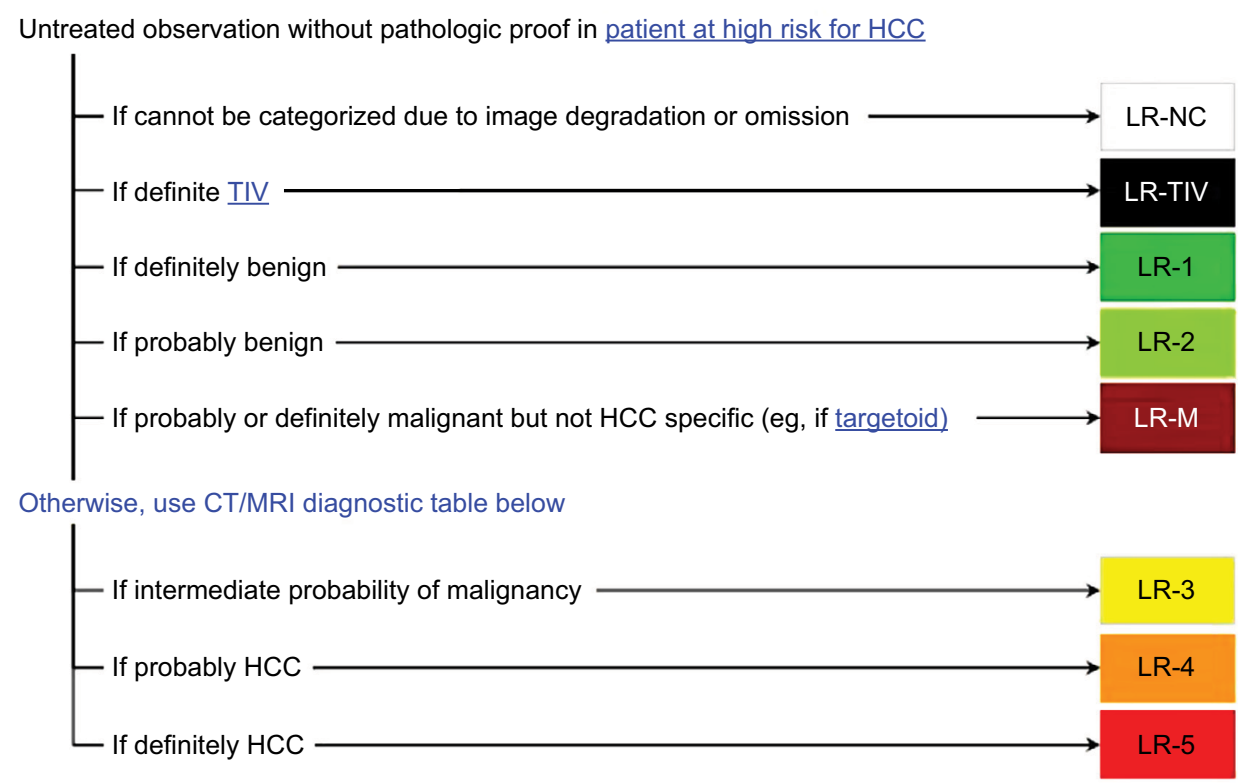

CT/MRI diagnostic table

\begin{tabular}{|c|c|c|c|c|c|c|}
\hline \multirow{2}{*}{$\begin{array}{r}\text { APHE } \\
\text { Observation size }(\mathrm{mm})\end{array}$} & & \multicolumn{2}{|c|}{ No APHE } & \multicolumn{3}{|c|}{ Nonrim APHE } \\
\hline & & $<20$ & $\geq 20$ & $<10$ & $10-19$ & $\geq 20$ \\
\hline \multirow{3}{*}{$\begin{array}{l}\text { Count additional major features: } \\
\text { - Enhancing "capsule" } \\
\text { - Nonperipheral "washout" } \\
\text { - Threshold growth }\end{array}$} & None & LR-3 & LR-3 & LR-3 & LR-3 & LR-4 \\
\hline & One & LR-3 & LR-4 & LR-4 & LR-4 & LR-5 \\
\hline & $\geq$ Two & LR-4 & LR-4 & LR-4 & LR-5 & LR-6 \\
\hline
\end{tabular}

LR-4 Observations in this cell are categorized based on one additional major feature:

- LR-4 - if enhancing "capsule"

- LR-5 - if nonperipheral "washout" OR threshold growth

If unsure about the presence of any major feature: characterize that feature as absent

Figure I LI-RADS CT/MRI diagnostic algorithm and table v20I8.

Notes: Reproduced with permission from American College of Radiology. Liver Imaging Reporting and Data System version 2018 core. Available from: https://www.acr. org/Clinical-Resources/Reporting-and-Data-Systems/LI-RADS. ${ }^{16}$

Abbreviations: ACR, American College of Radiology; APHE, Arterial phase hyperenhancement; HCC, hepatocellular carcinoma; LI-RADS, Liver Imaging Reporting and Data System; LR-NC, LR-noncategorizable; TIV, tumor in vein. 
technological advances. ${ }^{10}$ The major changes to LI-RADS for each update are enumerated in the following sections.

\section{LI-RADS v20I I}

The initial version of LI-RADS was released in 2011 with a standardized lexicon and five major categories for classifying observations in the liver: LR-1 (definitely benign), LR-2 (probably benign), LR-3 (intermediate probability for HCC), LR-4 (probably HCC), and LR-5 (definitely HCC). ${ }^{11}$

\section{LI-RADS v20 I 3}

The first major update to LI-RADS was released in 2013. It introduced a diagnostic table and imaging atlas. Modifications to the LR-5 category were made to achieve congruency between the Organ Procurement and Transplantation Network (OPTN) Class 5 and LR-5. Three new categories were formed: LR5 treated was congruent with the OPTN Class 5T category, OM (other malignancy) to account for malignant entities other than HCC that may occur in the liver, and LR5V for definitely $\mathrm{HCC}$ with the presence of tumor in vein (TIV).

\section{LI-RADS v20 I 4}

The 2014 LI-RADS update introduced material on hepatobiliary agents. The diagnostic algorithm was modified and some of the terminology was simplified. In particular, the designations $\mathrm{A}(\leq 19 \mathrm{~mm})$ and $\mathrm{B}(\geq 20 \mathrm{~mm})$, which split LR-4 and LR-5 categories based on observation size, were removed. ${ }^{12}$ A split cell was introduced into the algorithm for 10-19 mm observations with arterial phase hyperenhancement (APHE) and one additional major feature for HCC; observations in this cell with "washout" and visibility on antecedent screening ultrasound were assigned LR-5us as per the 2011 AASLD guidelines, ${ }^{13}$ observations in this cell meeting criteria for threshold growth were assigned LR-5g for congruency with OPTN Class 5A-g, ${ }^{14}$ and all other observations in this cell were assigned LR-4. OM was changed to LR-M (probably or definitely malignant, but not specific for $\mathrm{HCC}$ ), in recognition that corresponding lesions were not always an "other malignancy" but could be HCC and sometimes even could be benign. The lexicon and atlas were refined and expanded.

\section{LI-RADS v20I7}

LI-RADS v2017 added new algorithms for US surveillance, CEUS diagnosis, and CT/MRI treatment response assessment. The category LR-noncategorizable (LR-NC) was added to describe observations that cannot be categorized due to image degradation or omission. LR-5V was renamed LR-TIV in recognition that a minority of TIV observations represent non-HCC malignancy. ${ }^{15}$ Additionally, the threshold growth definition was modified, and new explicit criteria for LR-M were introduced.

\section{LI-RADS v2018}

The 2018 update achieved a major milestone by unifying LI-RADS and AASLD. The process of unification required revision of the LR-5 category (Table 1). In particular, the requirement for visibility at antecedent US for LR-5 designation for 10-19 mm observations with nonrim APHE and "washout" was removed. The LR-5 nomenclature was simplified by removal of the -us and - $g$ qualifiers. Additionally, the LI-RADS definition of threshold growth was also simplified to match that of the United Network for Organ Sharing and the Organ Procurement and Transplantation Network (UNOS-OPTN). ${ }^{16}$

\section{US LI-RADS}

\section{Overview}

Ultrasound is the most commonly used method for surveillance in patients at risk for HCC. It has the benefit of being a noninvasive, accessible, safe, and low-cost screening tool for HCC. In a meta-analysis of 15 scientific studies on HCC surveillance in patients with cirrhosis, sonography had a pooled sensitivity of $47 \%$ for early-stage cancer detection. ${ }^{17}$

Table I Major changes to CT/MRI LI-RADS v20I8

\begin{tabular}{|l|l|}
\hline \multicolumn{2}{|l|}{ Major changes to CT/MRI LI-RADS v20 I 8* } \\
\hline Type of change & Description \\
\hline Substantive & $\begin{array}{l}\text { Requirement of visibility on antecedent US for LR-5 designation of I0-I9 mm observations with nonrim APHE and } \\
\text { "washout" removed }\end{array}$ \\
\hline Substantive & OPTN definition of threshold growth adopted: $\geq 50 \%$ size increase of a mass in $\leq 6$ months \\
\hline Nomenclatural & -us removed as LR-5 qualifier \\
\hline Nomenclatural & -g removed as LR-5 qualifier \\
\hline
\end{tabular}

Note: *No changes made to CEUS LI-RADS or ultrasound LI-RADS

Abbreviations: APHE, arterial phase hyperenhancement; CEUS, contrast-enhanced ultrasound; LI-RADS, Liver Imaging Reporting and Data System; OPTN, Organ Procurement and Transplantation Network. 
Two prospective, randomized controlled trials in East Asia have shown that ultrasound surveillance decreased HCCrelated mortality by $31 \%-37 \% .{ }^{18,19}$ However, until now, there has been a lack of standardization of ultrasound interpretation and reporting. An ACR-endorsed working group developed a new LI-RADS algorithm specific to the interpretation of HCC screening and surveillance by ultrasound (US LI-RADS). ${ }^{20}$ Standardization of ultrasound technique and radiology reporting in high-risk individuals should improve communication between physicians and unify surveillance algorithms at different institutions, as accomplished by the CR/MRI LI-RADS diagnostic algorithm..$^{20}$ The appropriate patient population for the application of US LI-RADS is listed in Table 2. The primary at-risk population includes patients with cirrhosis from any etiology. The benefit of surveillance is unknown in adults with nonalcoholic steatohepatitis or hepatitis $\mathrm{C}$ infection in the absence of cirrhosis; however, these patients may be included in surveillance populations depending on regional practice guidelines. Patients with decompensated Child-Pugh $\mathrm{C}$ cirrhosis are excluded due to limited life expectancy unless the patient is a liver transplant candidate;

Table 2 The inclusion and exclusion criteria of LI-RADS (CT, MRI, CEUS, and ultrasound surveillance)

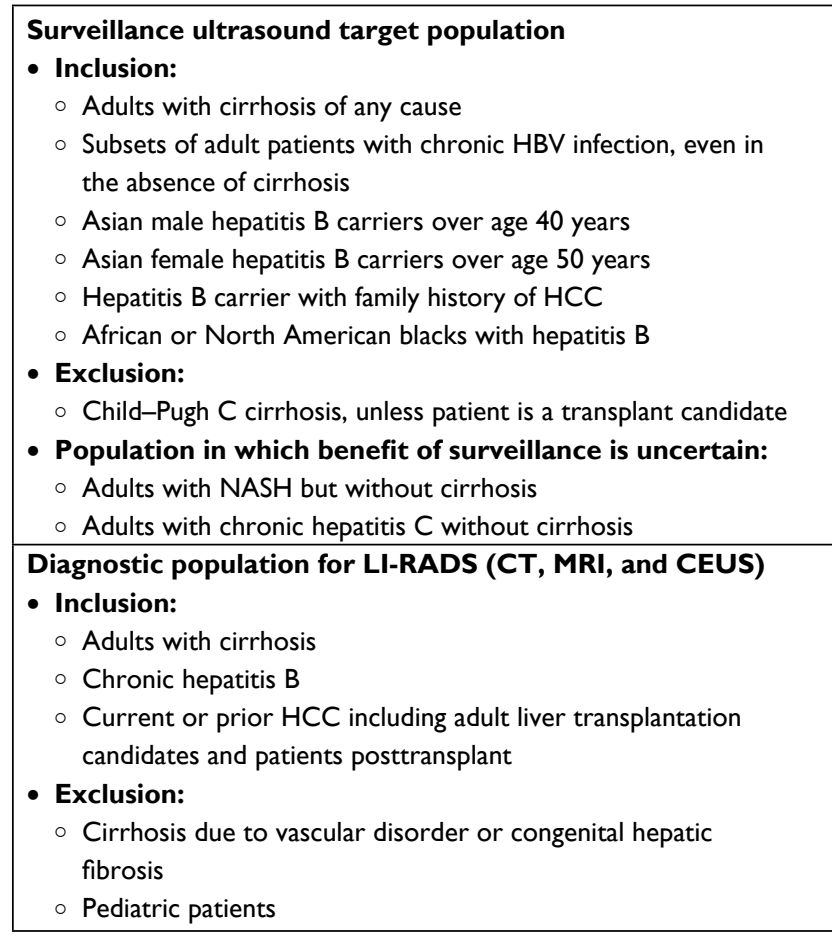

Notes: Target populations for screening and surveillance and target population for diagnostic imaging with CT, MRI, or CEUS. Guidelines based on the American Association for Study of Liver Diseases (AASLD).'

Abbreviations: CEUS, contrast-enhanced ultrasound; HCC, hepatocellular carcinoma; HPV, hepatitis B virus; LI-RADS, Liver Imaging Reporting and Data System; NASH, nonalcoholic steatohepatitis. however, the best strategy for HCC surveillance in transplant candidates is currently unclear.

The US LI-RADS algorithm includes two components: 1) an ultrasound category (Figure 2) and 2) visualization score (Figure 3). No changes to US LI-RADS were introduced in 2018 .

\section{Ultrasound category}

The study category, which is applied to the entire study as opposed to individual observations, determines management and potential need for further characterization of observations with a contrast-based study. ${ }^{20}$ The three categories are; 1) US-1: Negative, defined as no suspicious sonographic findings of HCC that warrant further evaluation. This includes the absence of any focal lesions or the presence of only definitely benign findings, such as focal fatty sparing, simple cyst, or previously confirmed hemangioma. The recommended management for category 1 is continued routine surveillance every 6 months. $^{20,21}$ 2) US-2: Subthreshold, defined as the presence of one or more focal lesions, all $<10 \mathrm{~mm}$, that are not definitely benign. The recommended management of US-2 involves close follow-up with short-interval ultrasound every 3-6 months to identify growth beyond the $1 \mathrm{~cm}$ threshold, in which case, further characterization with a contrast-based study is warranted. ${ }^{20,21}$ If an observation in an US-2 study is stable in size for 2 years or greater, the patient can return to routine 6-month surveillance. 3) US-3: Positive, defined as the presence of one or more observations $\geq 10 \mathrm{~mm}$ and not definitely benign. Such observations warrant further characterization with contrast-enhanced imaging. Examples include solid nodules $\geq 10 \mathrm{~mm}$ of any echogenicity (Figure 4), a new thrombus in a vein (whether considered bland thrombus or TIV), or focal parenchymal distortion $\geq 10 \mathrm{~mm}$ in size (Figure 5). The latter indicates the possibility of diffuse or infiltrative subtype of HCC, and it is defined by one or more of the following sonographic findings: ill-defined area of heterogeneity, refractive edge shadowing, loss of normal hepatic architecture, and distortion of vessels. The management for US-3 is further characterized with multiphase contrast-enhanced CT, MRI, or CEUS..$^{20,21}$

\section{Visualization score}

The visualization score is assigned to each examination based on technical quality and study limitations and conveys the expected sensitivity of the examination for detection of liver lesions. Multiple extrinsic and intrinsic factors can affect the quality of ultrasound visualization of the liver parenchyma including the patient body habitus, obscuration of the liver 


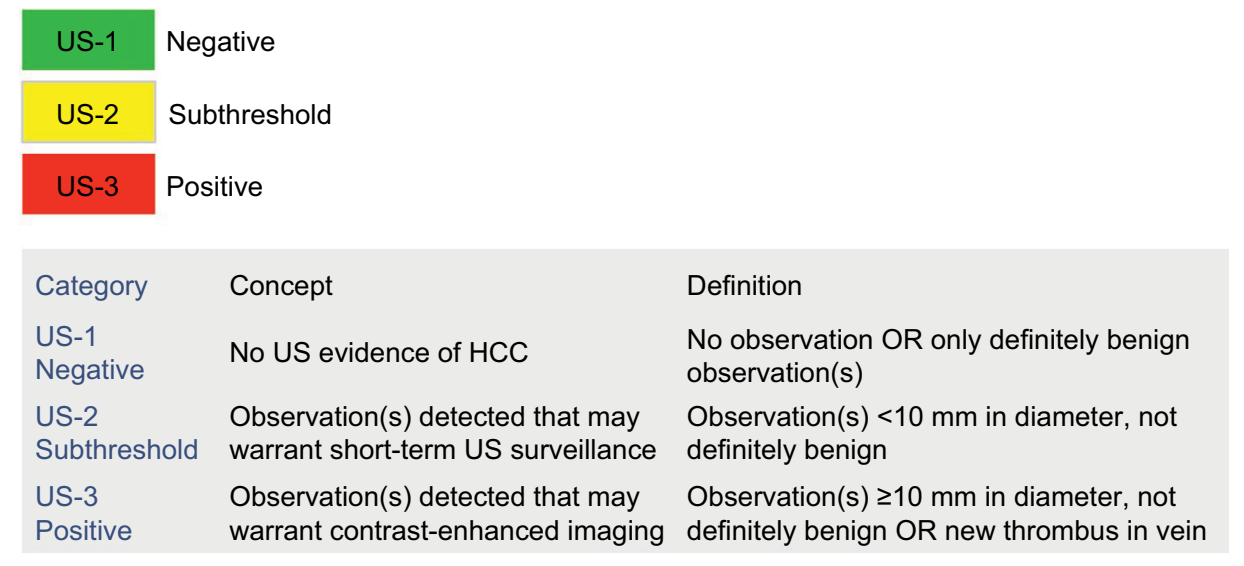

Figure 2 US LI-RADS US category.

Notes: Reproduced with permission from American College of Radiology. Ultrasound LI-RADS v20I7. https://www.acr.org/Clinical-Resources/Reporting-and-Data-Systems/ LI-RADS/Ultrasound-LI-RADS-v2017. ${ }^{21}$

Abbreviations: ACR, American College of Radiology; HCC, hepatocellular carcinoma; LI-RADS, Liver Imaging Reporting and Data System; US, ultrasound.

\begin{tabular}{|c|c|c|}
\hline A & \multicolumn{2}{|c|}{ No or minimal limitations } \\
\hline B & \multicolumn{2}{|c|}{ Moderate limitations } \\
\hline C & \multicolumn{2}{|c|}{ Severe limitations } \\
\hline Score & Concept & Examples \\
\hline $\begin{array}{l}\text { A. No or } \\
\text { minimal } \\
\text { limitations }\end{array}$ & $\begin{array}{l}\text { Limitations if any are } \\
\text { unlikely to meaningfully } \\
\text { affect sensitivity }\end{array}$ & $\begin{array}{l}\text { Liver homogeneous or minimally heterogeneous } \\
\text { Minimal beam attenuation or shadowing } \\
\text { Liver visualized in near entirety }\end{array}$ \\
\hline $\begin{array}{l}\text { B. Moderate } \\
\text { limitations }\end{array}$ & $\begin{array}{l}\text { Limitations may obscure } \\
\text { small masses }\end{array}$ & $\begin{array}{l}\text { Liver moderately heterogeneous } \\
\text { Moderate beam attenuation or shadowing } \\
\text { Some portions of liver or diaphragm not visualized }\end{array}$ \\
\hline $\begin{array}{l}\text { C. Severe } \\
\text { limitations }\end{array}$ & $\begin{array}{l}\text { Limitations significantly } \\
\text { lower sensitivity for focal } \\
\text { liver lesions }\end{array}$ & $\begin{array}{l}\text { Liver severely heterogeneous } \\
\text { Severe beam attenuation or shadowing } \\
\text { Majority }(>50 \%) \text { of liver not visualized } \\
\text { Majority }(>50 \%) \text { of diaphragm not visualized }\end{array}$ \\
\hline
\end{tabular}

Figure 3 US LI-RADS visualization score.

Notes: Reproduced with permission from American College of Radiology. Ultrasound LI-RADS v20I7. https://www.acr.org/Clinical-Resources/Reporting-and-Data-Systems/ LI-RADS/Ultrasound-LI-RADS-v2017.21

Abbreviations: ACR, American College of Radiology; LI-RADS, Liver Imaging Reporting and Data System.

by lung or bowel gas, a patient's inability to hold their breath or hold still, and hepatic parenchymal heterogeneity or sound attenuation due to fibrosis/cirrhosis or steatosis. ${ }^{20}$ The three scores are as follows: 1) Visualization A: No or minimal limitations, where the liver is visualized in its near entirety and there is little or no compromise on the sensitivity of detection of parenchymal masses. 2) Visualization B: Moderate limitations, which may decrease sensitivity of detection of small masses. Examples include moderate hepatic heterogeneity or difficult visualization of small portions of the liver. 3) Visualization C: Severe limitations, which may markedly lower the sensitivity for detection of liver observations. These limitations include severe parenchymal heterogeneity, substantial beam attenuation, which results in nonvisualization of large portions of the liver and diaphragm, or other factors that limit visualization of $>50 \%$ of the liver or diaphragm. ${ }^{20,21}$ It is important to note that currently the visualization score does not impact management recommendations - this is an active area of research, and as data and experience are collected, future versions of US LI-RADS may incorporate the visualization score into management recommendations.

\section{LI-RADS diagnostic population}

The criteria for the population on which LI-RADS can be applied for diagnosis, as opposed to surveillance, was introduced with v2017 and is carried over unchanged in 


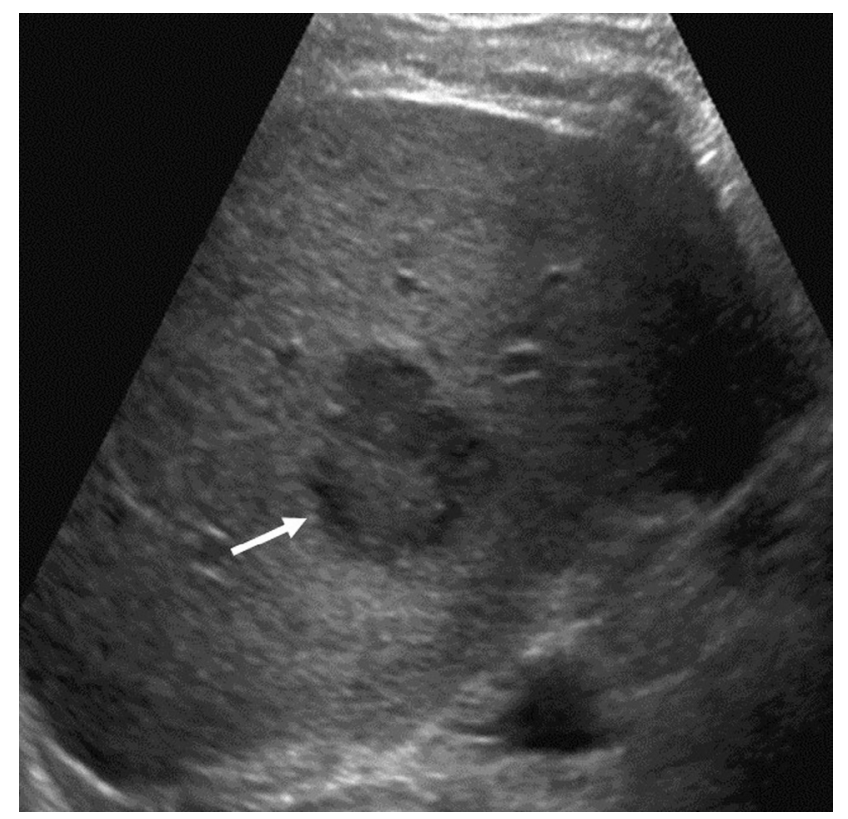

Figure 4 US LI-RADS Category 3 observation in a 59-year-old male with hepatitis $C$ cirrhosis undergoing US surveillance.

Notes: Sagittal US image shows a $3.6 \mathrm{~cm}$ solid hypoechoic observation with lobulated margins in segment 6 . This patient requires contrast-based studies; CEUS, CECT, or CEMRI to further characterize the lesion.

Abbreviations: CEUS, contrast-enhanced ultrasound; LI-RADS, Liver Imaging Reporting and Data System; US, ultrasound.

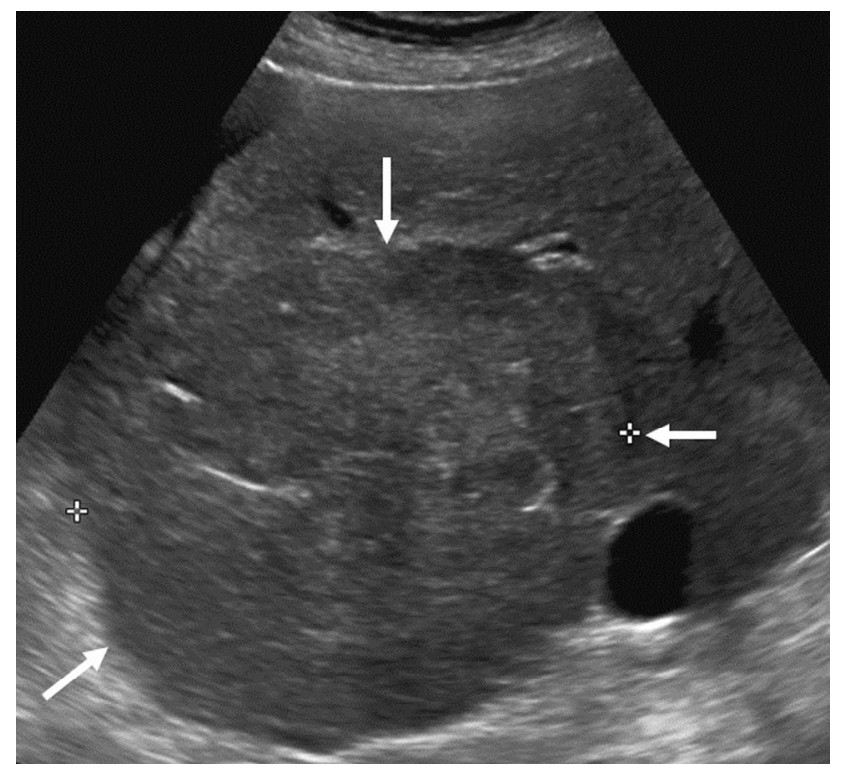

Figure 5 US LI-RADS Category 3 observation in a 70-year-old female with cryptogenic cirrhosis undergoing US surveillance.

Notes: Transverse US image shows a large area of heterogeneity (arrows) distinctive from background liver, shown to represent an HCC with infiltrative appearance on a diagnostic CEUS (not shown).

Abbreviations: CEUS, contrast-enhanced ultrasound; HCC, hepatocellular carcinoma; LI-RADS, Liver Imaging Reporting and Data System; US, ultrasound.

v2018. The inclusion criteria includes patients with cirrhosis, patients with chronic hepatitis $\mathrm{B}$ viral infection, and patients with current or prior HCC, including adult liver transplantation candidates and patients posttransplant. ${ }^{15,22}$ The exclusion criteria include cirrhosis due to either vascular disorders or cirrhosis due to congenital hepatic fibrosis. ${ }^{22-24}$ Vascular disorders leading to cirrhosis often have a large number of arterialized benign nodules resembling HCC, which can cause diagnostic confusion and reduce the diagnostic specificity. LI-RADS was not validated for use with the pediatric population, and as such, patients under 18 years old are excluded from the LI-RADS diagnostic population. ${ }^{15}$

\section{CT/MRI LI-RADS CT/MRI LI-RADS diagnostic categories}

Although no new categories have been introduced in version 2018, the LR-5 category was modified to be congruent with the AASLD practice guidelines. A brief review of the major imaging features, ancillary imaging features, and categories of LI-RADS is provided below, highlighting the modifications of the LR-5 category in version 2018.

\section{Major imaging features used in CT/MRI LI-RADS}

Five major imaging features are used to assign LR-3 through LR-5 categories for observations seen in patients at risk for HCC. Their presence should be unequivocal to maintain high specificity for HCC. ${ }^{25}$ The goal of standardization of definitions of these features is to encourage consistent application and interpretation, ultimately resulting in more consistent patient care, clearer education, and more rigorous and reproducible research. ${ }^{25}$

\section{Nonrim APHE}

This feature is defined as nonrim enhancement of an observation in the arterial phase that is unequivocally greater than the background liver tissue (Figure 6). It reflects the process of angiogenesis, which is a key component of $\mathrm{HCC}$ pathogenesis. ${ }^{26}$ This feature is considered present if either the entire observation or only a portion is hyperenhancing. The LR-5 category can only be assigned to observations with unequivocal nonrim APHE (and not simply vascular shunts with no correlation on other sequences; these are considered LR-2 or -3 observations), which is consistent with UNOS and OPTN criteria. ${ }^{27}$ Rim APHE is not a major feature of HCC, but would instead prompt assigning the LR-M category. ${ }^{27}$

\section{Nonperipheral "washout"}

The term nonperipheral washout appearance or "washout" is a perceived temporal reduction in enhancement of an observation relative to surrounding liver parenchyma from 


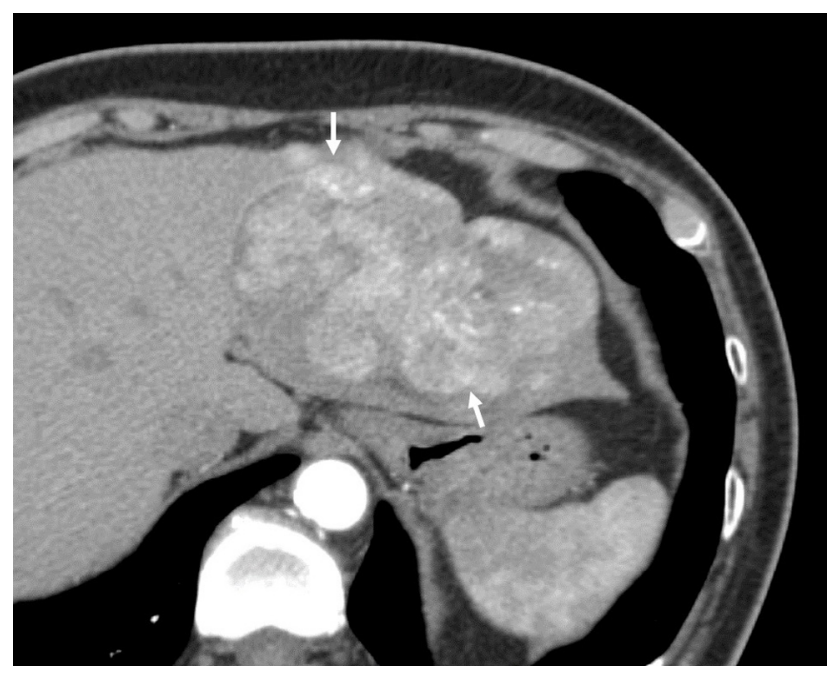

Figure 6 Seventy-one-year-old female demonstrating nonrim arterial phase hyperenhancement.

Notes: Contrast-enhanced CT shows a large mass (arrows) in the left hepatic lobe, partially exophytic, demonstrating heterogeneous arterial phase hyperenhancement. Posthepatectomy pathology confirmed well-differentiated hepatocellular carcinoma.

an earlier to later phase. ${ }^{28}$ This feature may be applied to any enhancing observation even in the absence of APHE. ${ }^{16}$ Although not fully understood, "washout" has been attributed to the lower extracellular volume of a tumor compared with background liver, which manifests as relative hypoenhancement in the postarterial extracellular phases. "Washout" can be assessed in the portal venous or delayed phase if an extracellular contrast agent is administered with MRI or CT. When using gadoxetate disodium, "washout" can only be assessed on the portal venous phase and cannot be reliably evaluated on the transitional or hepatobiliary phases because the background liver is changing as the hepatocytes take up the contrast medium..$^{29}$ One of the most reliable imaging hallmarks of $\mathrm{HCC}$ is the presence of nonrim APHE in combination with nonperipheral washout appearance. ${ }^{30}$ As opposed to nonperipheral "washout," peripheral washout is characteristic of intrahepatic cholangiocarcinoma (iCCA) and other non-HCC malignancies, hence is used as a criterion for assigning an LR-M category. ${ }^{15,27}$

\section{Enhancing "Capsule"}

"Capsule appearance" or "capsule" is defined as a uniform, sharp, smooth rim of hyperenhancement around most or all of an observation that is unequivocally thicker or more conspicuous than fibrotic tissue surrounding cirrhosis-related background liver nodules on portal venous, delayed, or transitional phases. The term, capsule appearance or "capsule," is preferred because its visualization does not always correlate with a pathologically identified true capsule. ${ }^{31,32}$ The degree of enhancement typically increases from early to later phases, reflecting the slow flow of intracapsular vessels. If a "capsule" is nonenhancing or visible only on unenhanced images, it is considered an ancillary feature "favoring HCC in particular," rather than a major LI-RADS feature. ${ }^{16}$ The capsule appearance is not to be confused with "corona enhancement" defined as periobservational enhancement in the late arterial phase or early portal venous phase. Corona enhancement is an ancillary feature favoring malignancy (but not specific for HCC). ${ }^{15}$

\section{Observation size}

As the shape of observations may be ovoid or irregular, the term size was adopted instead of diameter, which by definition, applies only to circles or spheres. ${ }^{27}$ Size is defined as the largest outer-edge-to-outer-edge dimension of an observation, including the capsule if present. ${ }^{15}$ Accurate measurement of observation size is important as it influences the staging of $\mathrm{HCC}$ and plays a role in determining transplant eligibility. ${ }^{33}$ It is also used for the assessment of threshold growth during follow-up imaging. The reader should measure observation size on the phase or sequence in which the margins are clearest. However, measurement during the arterial phase or diffusion-weighted imaging should be avoided because arterial phase measurement may overestimate size due to differences in timing of the arterial phase between scans or inadvertent inclusion of corona enhancement and the diffusion-weighted images tend to have anatomic distortion, which can affect measurements. ${ }^{16}$

\section{Threshold growth}

The definition of threshold growth was revised for v2018 to be consistent with definitions endorsed by OPTN. It is now defined as $\geq 50 \%$ increase in size of a mass in $\leq 6$ months. ${ }^{16}$ An unequivocal increase in size that does not meet the definition of "threshold growth" is considered an ancillary feature "favoring malignancy in general, not HCC in particular."16 Caution is required when applying growth as a diagnostic criterion for $\mathrm{HCC}$, as other malignant neoplasms (eg, iCCA and cHCC-CCA) can grow. ${ }^{25}$

\section{Ancillary imaging features used in CT/MRI LI-RADS}

Unlike the major features, the incorporation of ancillary imaging features, which are additional supportive features favoring malignancy or benignity, into the final category 
assignment is considered optional. ${ }^{15}$ It is up to the radiologist's clinical judgment to use these features to upgrade or downgrade an observation to refine the final category. ${ }^{34}$ Ancillary features can be used to upgrade (only up to LR-4) or downgrade an observation by one category only, regardless of how many ancillary features exist. However, ancillary features cannot upgrade an observation from LR-4 to LR-5 as these ancillary features do not currently have a high enough specificity for diagnosing HCC. ${ }^{15,27}$ Ancillary features are divided into three groups; 1) features favoring malignancy in general, 2) features favoring HCC in particular, and 3) features favoring benignity. ${ }^{15,34}$ If the features are conflicting, ie, demonstrating a mix of features favoring both malignancy and benignity, then the category should stay the same without change. ${ }^{27}$ Definitions of ancillary features are shown in Figure 7 and have not changed from v2017. Of note, these ancillary features apply to CT/MRI categorization and not to CEUS as there are different distinct features related to CEUS discussed later.

\section{CT/MRI LI-RADS categories CT/MRI LR-I to LR-5}

These represent the LI-RADS categories assigned to observations based on the probability of benignity vs malignancy determined by the presence of major and ancillary imaging features. ${ }^{15}$ LR-1 to LR-5 categories serve as a probabilistic scale that reflects the probability of benignity, malignancy in general, or HCC.

\section{CT/MRI LR-NC}

LR-NC is applied when there are considerable technical limitations to the image quality and therefore, assigning an LI-RADS category is not feasible. Based on the LI-RADS management algorithm, the radiologist may recommend repeat imaging at $\leq 3$ months if the cause of the technical limitation is resolvable or switch to an alternative imaging modality otherwise (eg, switching to CT from MRI if blooming artifact from an embolization coil obscures much of the liver). ${ }^{15,27}$

\section{CT/MRI LR-TIV (definitely malignant with TIV)}

LR-TIV is assigned when definite tumor invasion of a vein is observed regardless of whether there is an associated parenchymal mass. Although TIV is usually due to HCC, it can be caused by non-HCC malignancies such as iCCA and combined HCC-CCA tumors. ${ }^{15,27}$

\section{CT/MRI LR-M (malignancy not specific to $\mathrm{HCC}$ )}

This is assigned to probably or definitely malignant observations that do not meet the criteria for diagnosis of HCC.

\begin{tabular}{|c|c|}
\hline Ancillary features favoring malignancy & Ancillary features favoring benignity \\
\hline $\begin{array}{l}\text { Favoring malignancy in general, not HCC in } \\
\text { particular } \\
\text { - US visibility as discrete nodule } \\
\text { - Subthreshold growth } \\
\text { - Restricted diffusion } \\
\text { - Mild-moderate T2 hyperintensity } \\
\text { - Corona enhancement } \\
\text { - Fat sparing in solid mass } \\
\text { - Iron sparing in solid mass } \\
\text { - Transitional phase hypointensity } \\
\text { - Hepatobiliary phase hypointensity }\end{array}$ & $\begin{array}{l}\text { - Size stability }>2 \text { years } \\
\text { - Size reduction } \\
\text { - Parallels blood pool } \\
\text { - Undistorted vessels } \\
\text { - Iron in mass, more than liver } \\
\text { - Marked T2 hyperintensity } \\
\text { - Hepatobiliary phase isointensity }\end{array}$ \\
\hline $\begin{array}{l}\text { Favoring HCC in particular } \\
\text { - Nonenhancing "capsule" } \\
\text { - Nodule-in-nodule } \\
\text { - Mosaic architecture } \\
\text { - Blood products in mass } \\
\text { - Fat in mass, more than adjacent liver }\end{array}$ & \\
\hline
\end{tabular}

Figure 7 Ancillary imaging features used in LI-RADS CT/MRI.

Note: These ancillary features are supportive, and their use is optional. Reproduced with permission from American College of Radiology. Liver Imaging Reporting and Data System version 2018 core. Available from: https://www.acr.org/Clinical-Resources/Reporting-and-Data-Systems/LI-RADS.16

Abbreviations: ACR, American College of Radiology; HCC, hepatocellular carcinoma; LI-RADS, Liver Imaging Reporting and Data System; US, ultrasound. 
These lesions have a broad differential diagnosis that includes not only HCC but also metastases, iCCA, or combined tumors. ${ }^{15,35}$ This category was devised to preserve the specificity of the LR-5 category without loss of sensitivity for detection of malignancy.

\section{Assigning LI-RADS diagnostic category using CT/MRI}

Prior to applying the LI-RADS diagnostic algorithm, the radiologist should ensure the patient is at increased risk of developing HCC (as described above in LI-RADS diagnostic population). Selectively applying LI-RADS algorithms to at-risk populations is important in avoiding diagnostic errors and maintaining the high specificity of the LR-5 category. ${ }^{5}$ LR-5 (definitely HCC) carries a nearly 100\% specificity for $\mathrm{HCC}$, which eliminates the need for histopathological confirmation. The diagnostic algorithm also should not be applied to focal observations after locoregional therapy; the treatment response algorithm should be applied instead. ${ }^{36}$ After these initial checks, a simplified four-step approach to assigning LI-RADS categories can be performed, which is unchanged in v2018. ${ }^{15}$

\section{Step I:Apply CT/MRI LI-RADS diagnostic algorithm}

First, the interpreting radiologist should examine the study for quality and completeness in terms of necessary dynamic enhancement phases to assess whether assigning a diagnostic category is feasible. ${ }^{5,15,27}$ If key imaging phases are missing or obscured by artifact, then the observation should be assigned LR-NC.

Once the quality and completeness of the study is confirmed, the liver should be evaluated for the presence of TIV (Figure 8). If present, LR-TIV category should be assigned regardless of the visualization of a parenchymal observation. The reporting of LR-TIV should reflect the possible etiologies of the venous invasion as follows: 1) if TIV is contiguous with a targetoid parenchymal mass, report: "LR-TIV, may be due to non-HCC malignancy"; 2) if TIV is contiguous with an observation meeting LR-5 criteria, report: "LR-TIV, definitely due to HCC"; and 3 ) in all other scenarios, report: "LR-TIV, probably due to HCC." 5

After excluding the presence of TIV, the radiologist should consider the features of individual observations and determine if they are definitely (LR-1) or probably (LR-2) benign. ${ }^{15}$ Definitely benign observations (LR-1) include hemangiomas, cysts, confluent fibrosis, and focal fat deposition or sparing. Probably benign observations (LR-2) have high but not $100 \%$ probability of benignity, such as probable hemangioma, probable perfusion alterations, and $<20 \mathrm{~mm}$ distinctive nodules with no major or ancillary features favoring malignancy. ${ }^{37,38}$

If an observation does not meet the criteria for LR-1 or LR-2, then it should be determined if it meets the criteria for LR-M (Figure 9). LR-M is assigned to solid observation with a targetoid appearance (Figure 9) or with one or more of the following imaging features: infiltrative appearance, marked diffusion restriction, necrosis, or severe ischemia. ${ }^{5,35}$

If all the categories above are excluded after stepwise consideration, the LI-RADS CT/MRI diagnostic table (Figure 1) is applied and the observation is assigned a category LR-3, LR-4, or LR-5 based on the presence of major features. ${ }^{16}$ First, the observation should be assessed for the presence of nonrim APHE. Second, the size of the observation is measured to determine the correct column in the table. ${ }^{5,16,27}$ Observations without APHE are separated into $<20 \mathrm{~mm}$ or $\geq 20 \mathrm{~mm}$, while observations with nonrim APHE are divided into $<10 \mathrm{~mm}, 10-19 \mathrm{~mm}$, and $\geq 20 \mathrm{~mm}$. Note that LR-5 category is reserved for observations, which are both $10 \mathrm{~mm}$ or larger and demonstrate nonrim APHE. Finally, the number of major features present other than nonrim APHE is assessed to determine the appropriate row in the table..$^{5,16}$ The cell at the intersection between the selected column and row contains the correct LI-RADS category.

\section{Step 2:Apply ancillary features}

After assigning the initial LI-RADS category, the radiologist may elect to apply ancillary features to adjust the category as explained previously. 5,34

\section{Step 3:Apply tiebreaking rules}

If, after following the algorithm through the first two steps, there is still uncertainty between two categories (often due to uncertainty regarding the presence of one or more imaging features), the radiologist should then choose the category associated with lower diagnostic certainty. ${ }^{16,27}$ For example, if the radiologist is uncertain regarding the presence of TIV, then LR-TIV should not be assigned.

\section{Step 4: Perform a final check}

Finally, the radiologist should consider whether the assigned category seems appropriate based on their clinical judgment. If it is appropriate, then the category is finalized, and the 

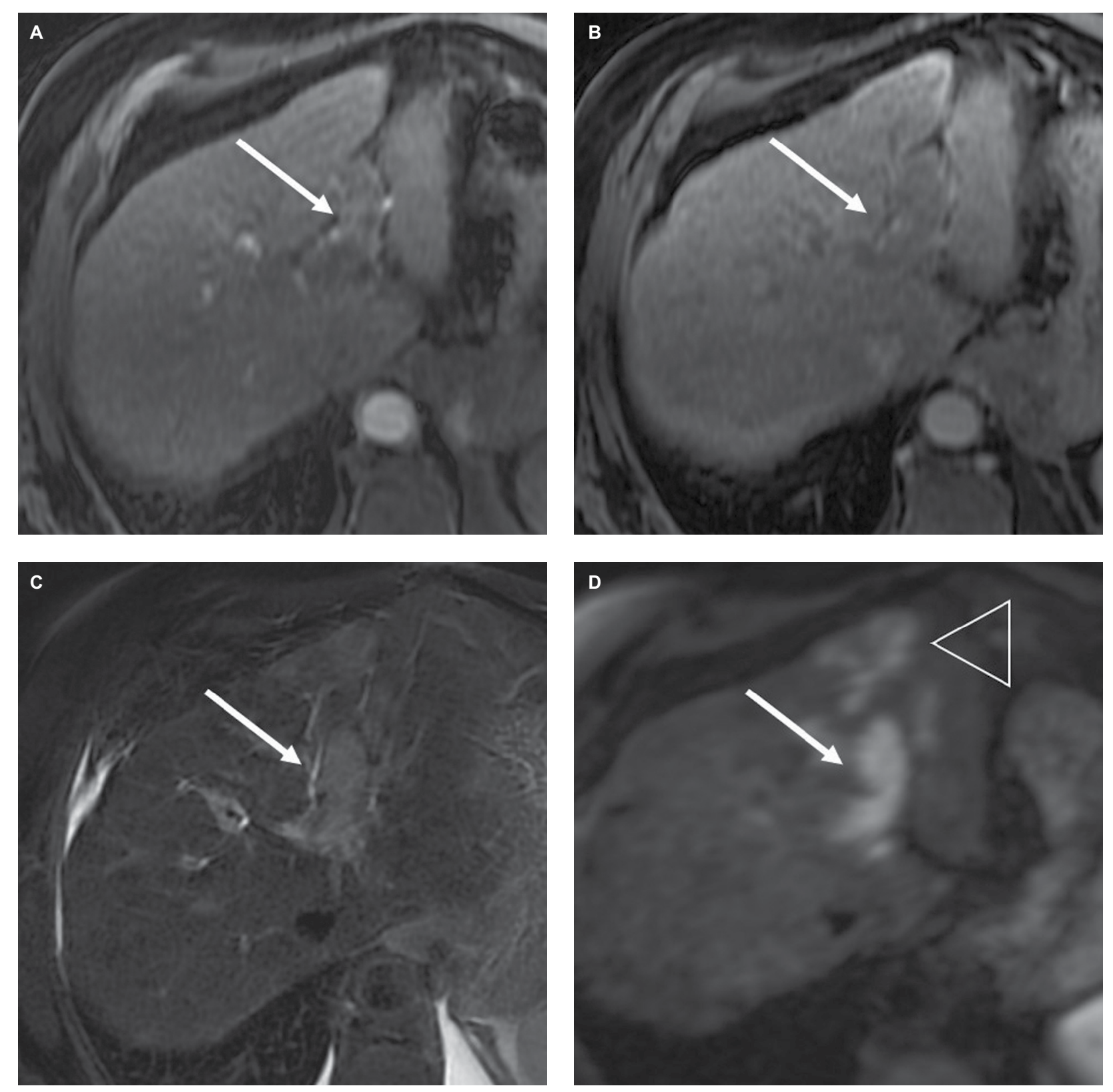

Figure 8 Fifty-eight-year-old man with cirrhosis (AFP=285 ng/mL) tumor in vein (TIV), probably due to hepatocellular carcinoma.

Notes: Gadobenate dimeglumine-enhanced MRI shows unequivocal enhancement of tissue (arrow) within the left portal vein, which meets the criterion for LR-TIV. Notice that the tissue expands the lumen of the left portal vein, (A) hyperenhances in the arterial phase, (B) appears to washout in the portal venous phase and has high signal intensity on (C) T2-weighted and (D) diffusion-weighted images. Involvement of the parenchyma by the tumor is more conspicuous on diffusion-weighted images (arrowhead in $\mathbf{D})$.

radiologist should move on to the next observation. If not, then the radiologist should reevaluate. Consultation with a colleague for a second opinion may be reasonable if doubt persists. $^{5}$

\section{CEUS LI-RADS}

\section{CEUS LI-RADS diagnostic categories}

The diagnostic categories of CEUS (Figure 10) follow the same general template of CT/MRI with some modifications to the criteria required to assign these categories. ${ }^{39}$

\section{CEUS LR-NC}

This category is usually assigned to observations where significant limitations of the technique or poor quality of the study due to external factors such as large body habitus would prevent assigning an appropriate category to the observation..$^{39,40}$

\section{CEUS LR-TIV}

This is assigned when there is unequivocal visualization of enhancing soft tissue in vein, regardless of visualization of 

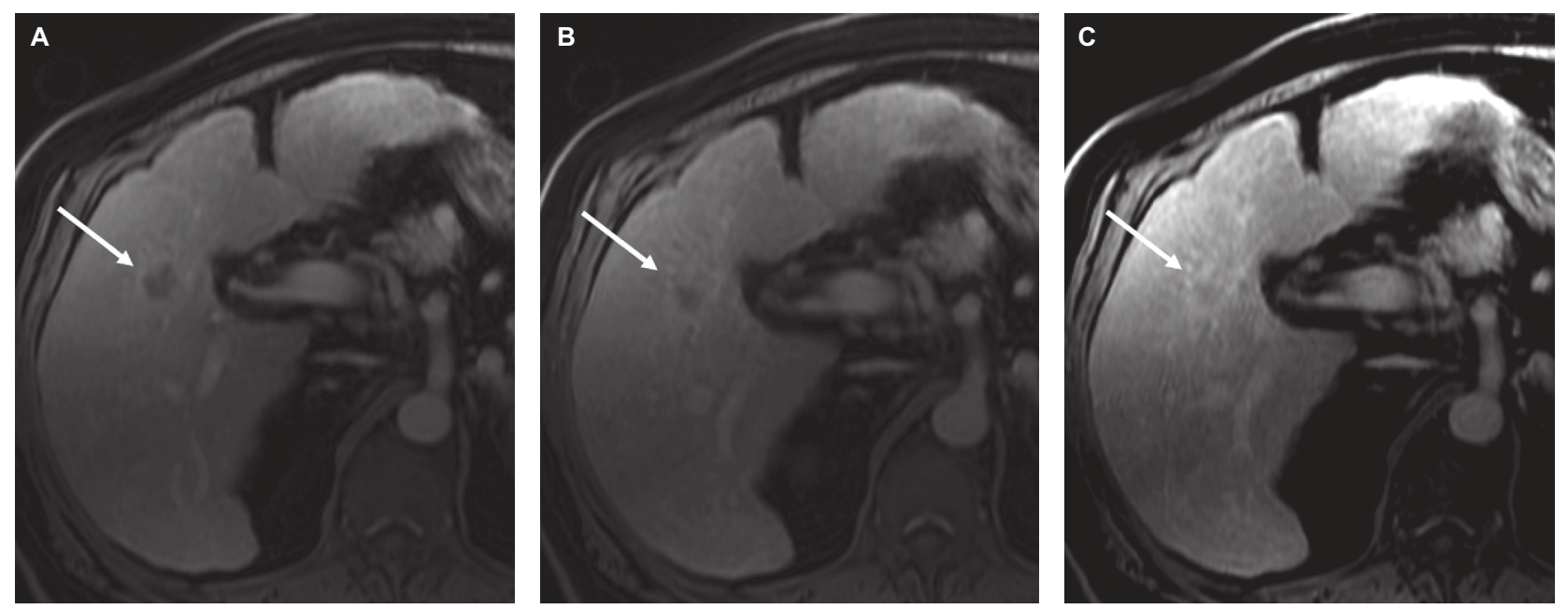

Figure 9 Sixty-one-year-old man with chronic hepatitis $C$ and cirrhosis.

Notes: A $2 \mathrm{~cm}$ observation in hepatic segment 5 shows (A) rim arterial phase hyperenhancement, (B) progressive delayed central enhancement on portal venous, and (C) delayed phase, corresponding to a targetoid appearance (LR-M). Biopsy confirmed intrahepatic cholangiocarcinoma. Arrow shows the enhancement pattern.

\section{CEUS LI-RADS ${ }^{\circledR} 2017$ Categories}

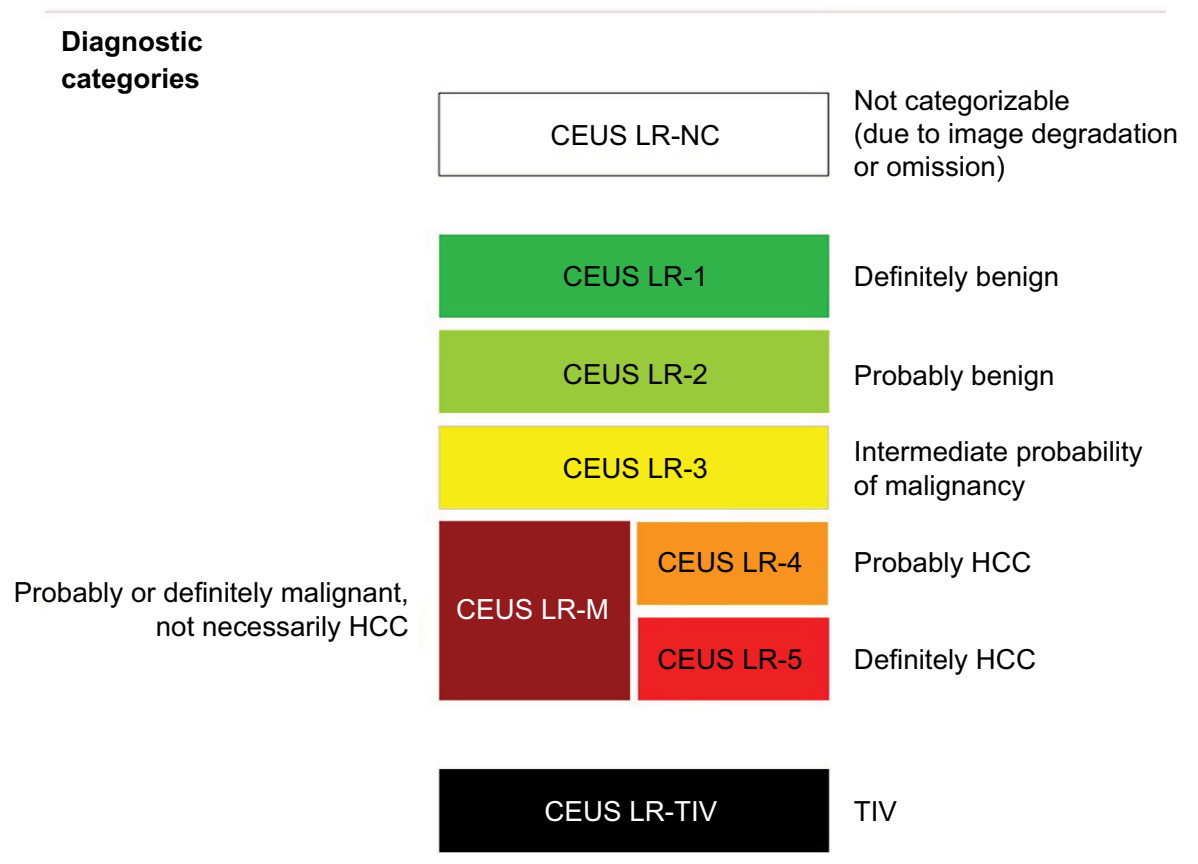

Figure 10 Diagnostic categories for CEUS LI-RADS.

Notes: Reproduced with permission from American College of Radiology. CEUS LI-RADS v2017. Available from: https://www.acr.org/Clinical-Resources/Reporting-andData-Systems/LI-RADS/CEUS-LI-RADS-v2017. ${ }^{39}$

Abbreviations: ACR, American College of Radiology; CEUS, contrast-enhanced ultrasound; HCC, hepatocellular carcinoma; LI-RADS, Liver Imaging Reporting and Data System; LR-NC, LR-noncategorizable; TIV, tumor in vein.

parenchymal mass (Figure 11). CEUS is particularly helpful in differentiation between TIV and bland thrombus depending on the time of microbubble contrast agent arrival to the vein, where early arrival (same as hepatic artery) favors TIV and arrival several seconds after hepatic artery enhancement would favor recanalized/nonocclusive bland thrombus. ${ }^{39}$ 

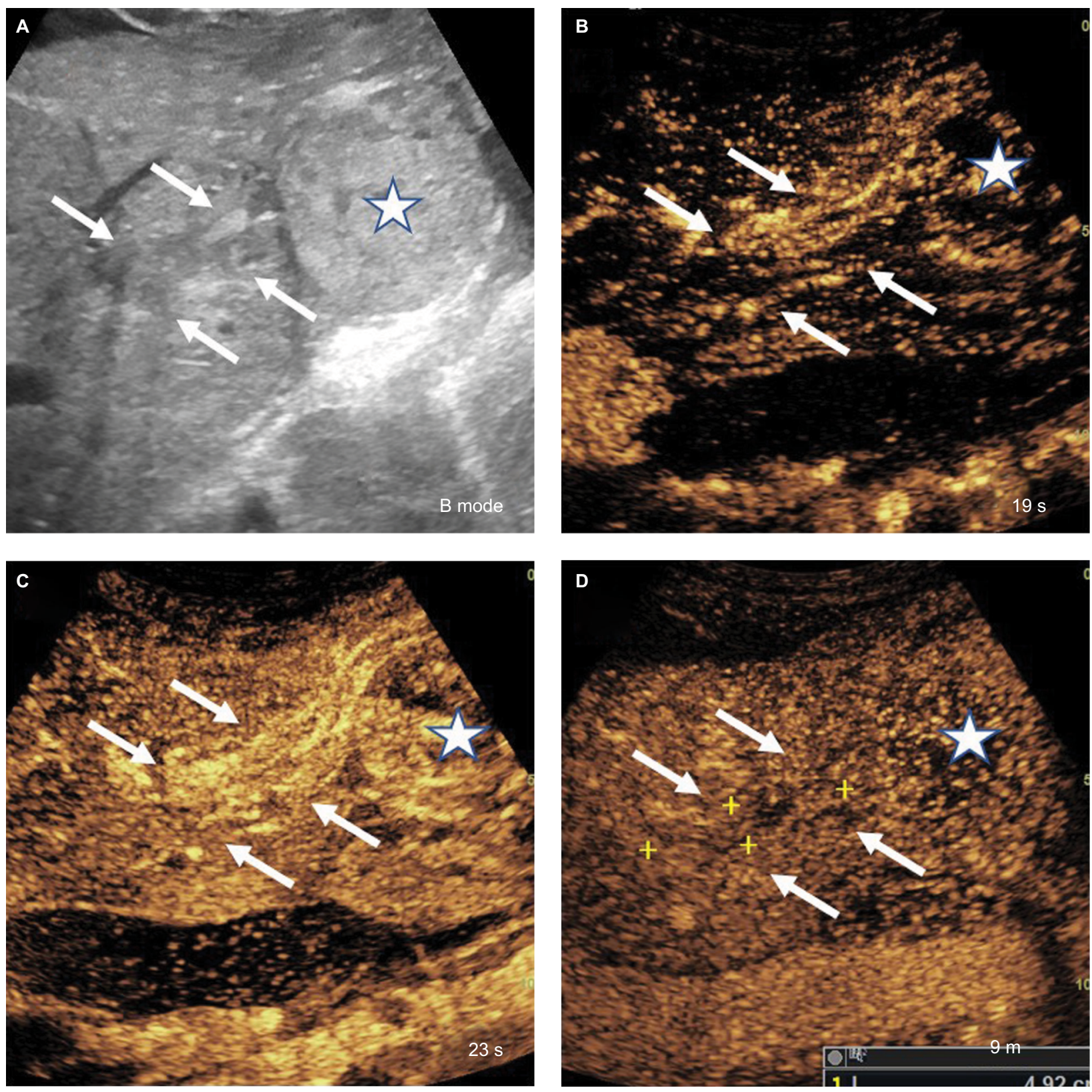

Figure II CEUS LI-RADS TIV.

Notes: (A) Soft tissue in the portal vein (arrows) contiguous with a parenchymal mass (star) on B-mode ultrasound. (B, C) Both soft tissue within the portal vein (arrows) and the mass (star) show arterial phase hyperenhancement and (D) mild washout in the late phase. CEUS LI-RADS TIV Criteria:

- Unequivocal arterial phase hyperenhancement and washout of soft tissue within the lumen of portal and/or hepatic veins.

- Must correspond on dual screen with mass in vein.

- Most LR-TIVs are HCC. Some are iCCA or cHCC-CCA.

- CEUS has been shown to have high sensitivity and specificity to diagnose TIV.

Abbreviations: CEUS, contrast-enhanced ultrasound; HCC, hepatocellular carcinoma; iCCA, intrahepatic cholangiocarcinoma; TIV, tumor in vein.

\section{CEUS LR-M}

This category is assigned to a probably or definitely malignant nodule, not particularly an HCC. The criteria for inclusion into LR-M are shown in Figure 12.

\section{CEUS LR-I to LR-5}

These are the same categories as CT/MRI LI-RADS and are assigned based on the probability of the observation being benign (LR-1 and LR-2) or malignant (LR-3, LR-4, and 


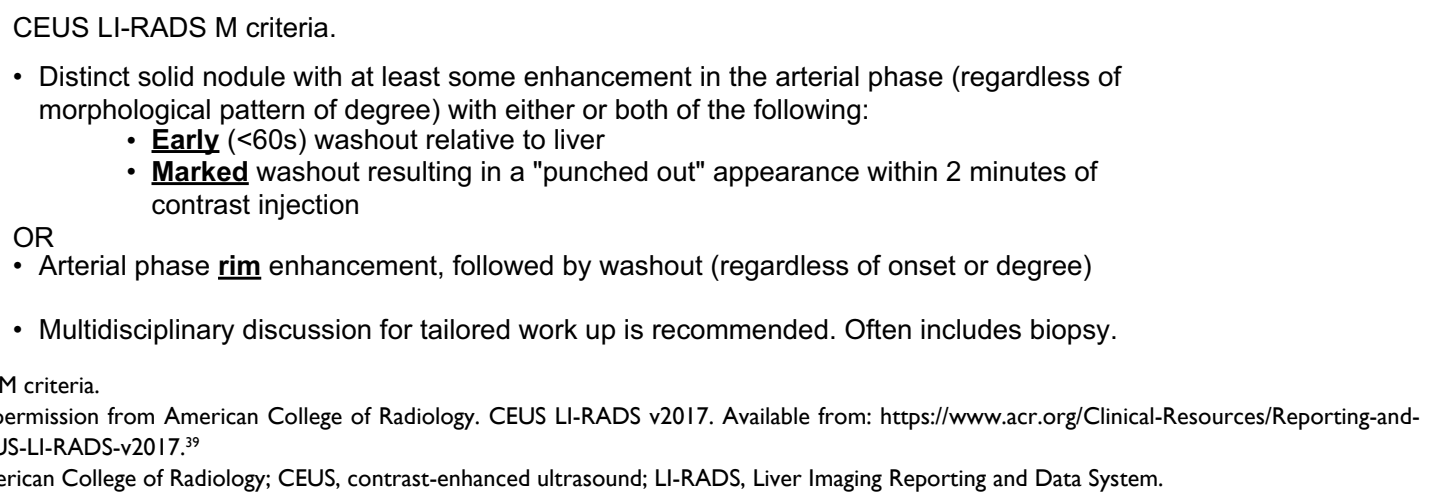

LR-5). The major difference lies in the number of major and ancillary features used to assign categories LR-3 to LR-5. ${ }^{39}$

\section{Major imaging features used in CEUS LI-RADS} Nonrim APHE

Similar to CT/MRI, nonrim APHE is one of the major features for CEUS LI-RADS and is considered present if demonstrated in either the entirety or just a portion of the nodule. In contrast to $\mathrm{CT} / \mathrm{MRI}$, CEUS or grayscale ultrasound does not visualize arterioportal shunts. ${ }^{40}$ Thus, in contradistinction to CT and MRI, most observations detectable by CEUS are true nodules, and the presence of nonrim APHE by itself prompts LR-4 categorization, regardless of nodule size. Of note, if the APHE on CEUS is peripheral, discontinuous, and globular, this is characteristic of a hemangioma. ${ }^{39}$

\section{Washout}

CEUS uses purely intravascular microbubble contrast agents, which makes CEUS washout a true washout and does not require using the terms washout appearance or "washout." 39 CEUS may be used to differentiate between typical HCC and other malignant lesions that show APHE by assessment of the onset (late vs early) and degree (mild vs marked) of washout. Early-onset washout is considered present when detected within 60 seconds of contrast agent injection, while late-onset washout is characterized when detected at or after the 60 -second mark (Figure 13A). ${ }^{40}$ Marked washout is considered present when the nodule is mostly devoid of contrast and is seen as "punched out" within 2 minutes after contrast injection (Figure 13B). Marked washout is one of the characteristics of LR-M category. Mild washout, however, is defined as a nodule demonstrating less degree of enhancement than the surrounding liver parenchyma but still possessing some degree of contrast enhancement. ${ }^{40}$ Nodules with mild washout may later appear as showing marked washout. Thus, if this occurred after 2 minutes, it should still be categorized as mild washout. HCCs usually show late-onset mild washout in contrast to non-HCC malignancies that demonstrate earlyonset and/or marked washout (Figure 13C). ${ }^{39}$

\section{Ancillary imaging features used in CEUS LI-RADS}

Ancillary features are applied less often in CEUS compared with CT/MRI. Similar to CT/MRI, they can be used to upgrade or downgrade the category of an observation; similarly, they cannot be used to upgrade LR-4 to LR-5. ${ }^{39}$ One advantage of CEUS over CT/MRI is the fewer number of ancillary features, which reduces the interpretive burden on the radiologist. Definitions of these features are shown in Figure 14. They are also divided into three categories, analogous to those used in CT and MRI. ${ }^{39}$

\section{Assigning LI-RADS diagnostic category using CEUS}

CEUS is used similar to contrast-enhanced CT and MRI for dynamic evaluation of lesions as well as surrounding blood flow in the form of APHE and washout. ${ }^{39,40}$ Limitations of CEUS are that it cannot be used for staging the entire liver, and it has not been validated for the assessment of treatment response. ${ }^{39,41}$ The population in which CEUS LI-RADS can be applied is the same as that of CT/MRI. CEUS is indicated for the characterization of observations $\geq 10 \mathrm{~mm}$ when detected on a surveillance ultrasound. It can also be used to detect APHE when evaluation with CT or MRI is not possible (or technically suboptimal). CEUS can be used to evaluate previously biopsied observations with inconclusive histology and to differentiate between TIV and bland thrombus. ${ }^{39}$ Although the key concepts and principles of CEUS categories (Figure 10) appear similar to those of CT/MRI, there are important differences that warrant separate diagnostic features and characterization algorithms. ${ }^{39,40}$ 

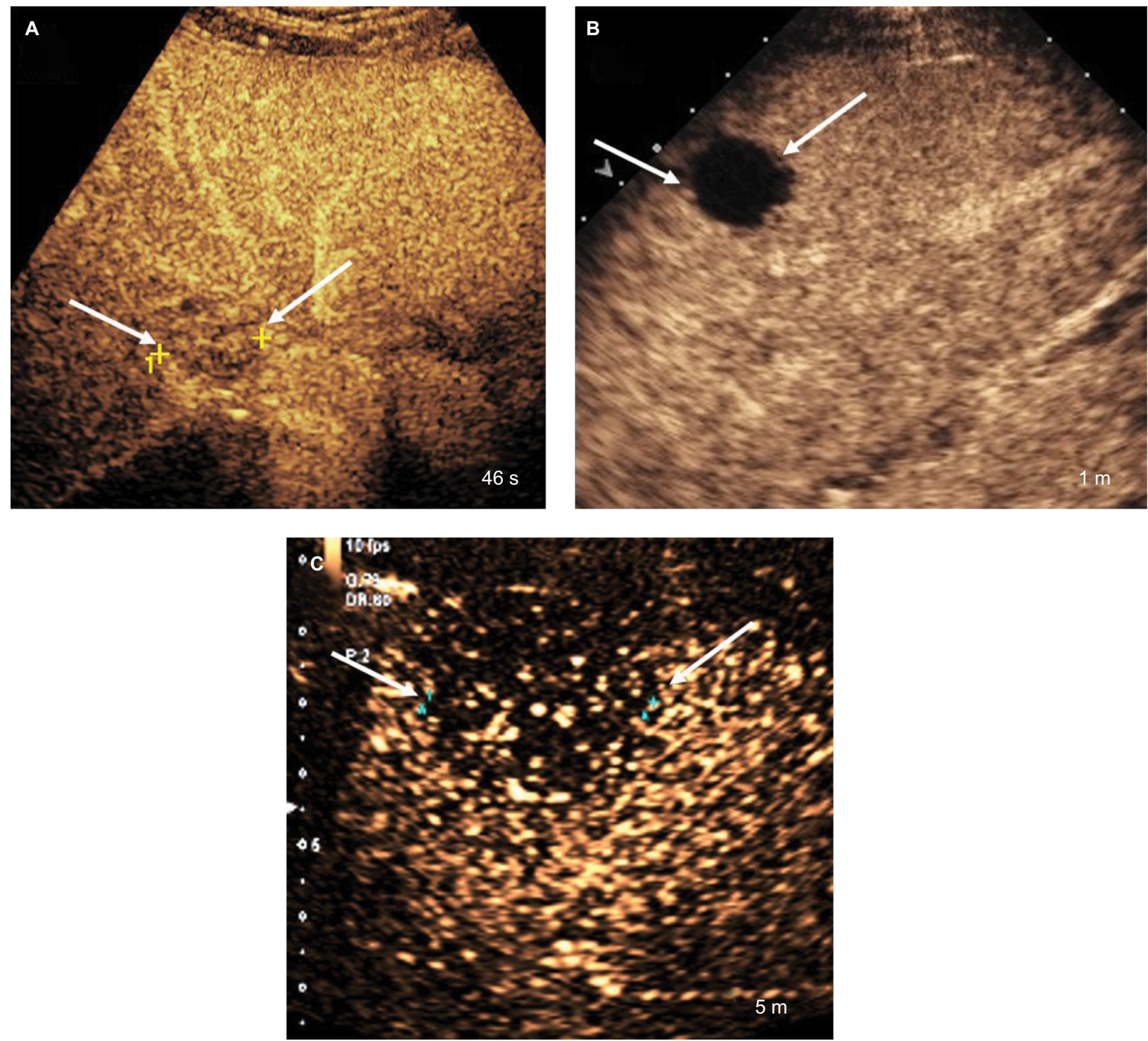

Figure 13 Washout.

Notes: (A) Early weak washout seen within I minute after contrast injection (CEUS LR-M feature). (B) Marked washout seen within 2 minutes after contrast injection (CEUS LR-M feature). (C) Late and mild washout seen >I minute after contrast injection (CEUS LR-5 feature).

Abbreviation: CEUS, contrast-enhanced ultrasound.

Ancillary features.

CEUS AFs favoring malignancy

Favoring malignancy in general, not $\mathrm{HCC}$ in

particular

- Definite growth

Favoring $\mathrm{HCC}$ in particular

- Nodule-in-nodule architecture

- Mosaic architecture

\section{CEUS AFs favoring benignity}

- Size stability $\geq 2$ years

- Size reduction

If unsure about presence of any ancillary feature: characterize that feature as absent

Figure 14 AFs used in CEUS LI-RADS.

Notes: Reproduced with permission from American College of Radiology. CEUS LI-RADS v2017. Available from: https://www.acr.org/Clinical-Resources/Reporting-andData-Systems/LI-RADS/CEUS-LI-RADS-v2017.39

Abbreviations: ACR, American College of Radiology; AF, ancillary feature; CEUS, contrast-enhanced ultrasound; HCC, hepatocellular carcinoma; LI-RADS, Liver Imaging Reporting and Data System. 


\section{Step I:Apply the CEUS LI-RADS diagnostic algorithm}

The steps to apply CEUS diagnostic algorithm (Figure 15) follow closely those of CT/MRI. Below a few distinctions are highlighted.

If a definitely benign lesion is eliminated from consideration, then an observation can be categorized as probably benign (LR-2) based on the following criteria; 1) distinct isoenhancing solid nodule $<10 \mathrm{~mm}, 2$ ) nonmass-like isoenhancing observation of any size while not being of typical fatty change/sparing appearance, and 3) LR-3 nodules with interval stability for at least 2 years. If an observation does not meet the LR-1 or LR-2 criteria, then it should be evaluated for the presence of LR-M criteria (Figure 12). On CEUS, these criteria include 1) early washout relative to background liver within 60 seconds of the contrast agent injection, 2) marked washout resulting in a punched-out appearance within 2 minutes of contrast agent injection, and 3) rim APHE followed by washout.

After exclusion of LR-M, CEUS LI-RADS diagnostic table should be applied and categories LR-3, LR-4, or LR-5 should be assigned to the observation based on size and the presence of major features. LR-4 category is assigned to observations that have high but not $100 \%$ probability of HCC. LR-5 category is only assigned to observations at least $10 \mathrm{~mm}$ or larger with nonrim APHE and mild late-onset washout. The CEUS diagnostic table is similar to the CT/MRI diagnostic table. One exception is that greater emphasis is placed on nonrim APHE because any observation with nonrim APHE is an arterialized nodule as described earlier. Another exception is that the characterization of washout is based on its timing and degree, not just on its presence. Figures 15-17

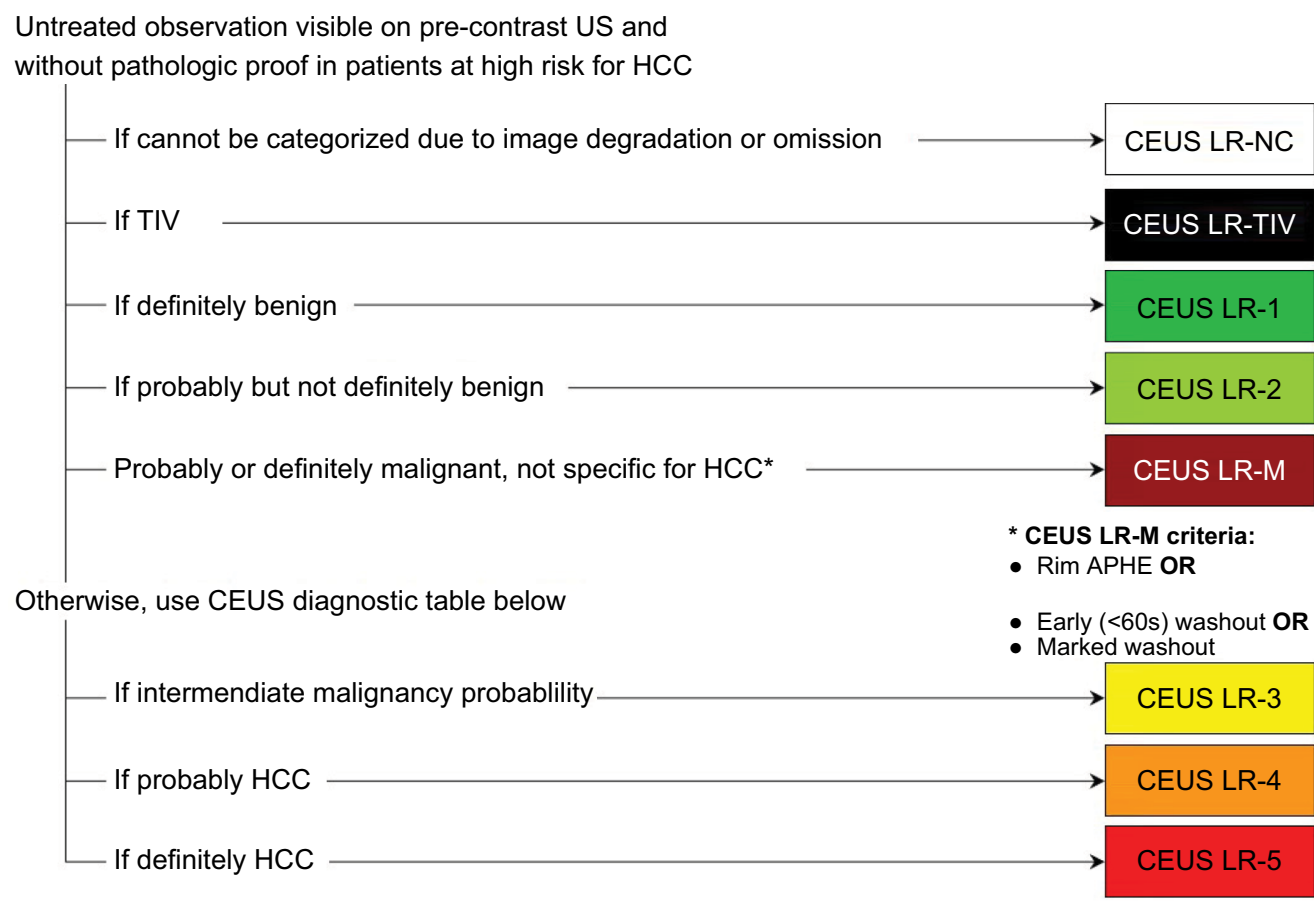

CEUS diagnostic table

\begin{tabular}{|c|c|c|c|c|}
\hline APHE & \multicolumn{2}{|c|}{ No APHE } & \multicolumn{2}{|c|}{ APHE ** } \\
\hline Nodule size (mm) & $<20$ & $\geq 20$ & $<10$ & $\geq 10$ \\
\hline No washout of any type & CEUS LR-3 & CEUS LR-3 & CEUS LR-3 & CEUS LR-4 \\
\hline Late and mild washout & CEUS LR-3 & CEUS LR-4 & CEUS LR-4 & CEUS LR-5 \\
\hline
\end{tabular}

Figure 15 CEUS LI-RADS diagnostic algorithm and table.

Notes: Reproduced with permission from American College of Radiology. CEUS LI-RADS v2017. Available from: https://www.acr.org/Clinical-Resources/Reporting-andData-Systems/LI-RADS/CEUS-LI-RADS-v2017. ${ }^{39}$

Abbreviations: ACR, American College of Radiology; APHE, arterial phase hyperenhancement; CEUS, contrast-enhanced ultrasound; HCC, hepatocellular carcinoma; LIRADS, Liver Imaging Reporting and Data System; ; LR-NC, LR-noncategorizable; TIV, tumor in vein; US, ultrasound. 

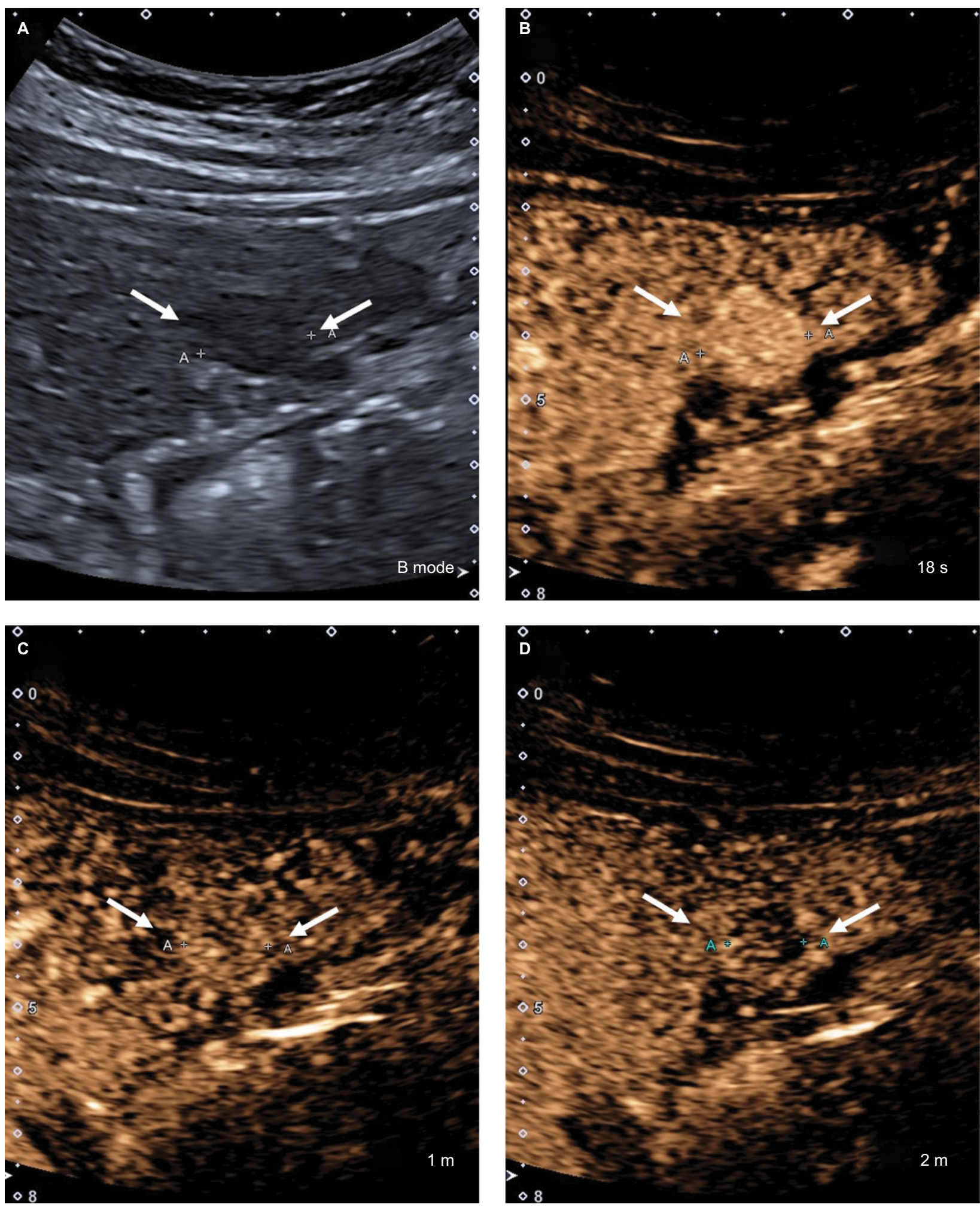

Figure 16 CEUS LR-5

Notes: (A) A $17 \mathrm{~mm}$ hypoechoic nodule on B-mode ultrasound. (B) The entire nodule shows hyperenhancement in the arterial phase. (C) At I minute, the nodule is isoenhancing compared with the surrounding liver parenchyma. (D) The nodule shows mild but definite hypoenhancement compared with the surrounding liver parenchyma at 2 minutes. This is late and mild washout. Arrows show the outline of the nodule.

Abbreviation: CEUS, contrast-enhanced ultrasound. 

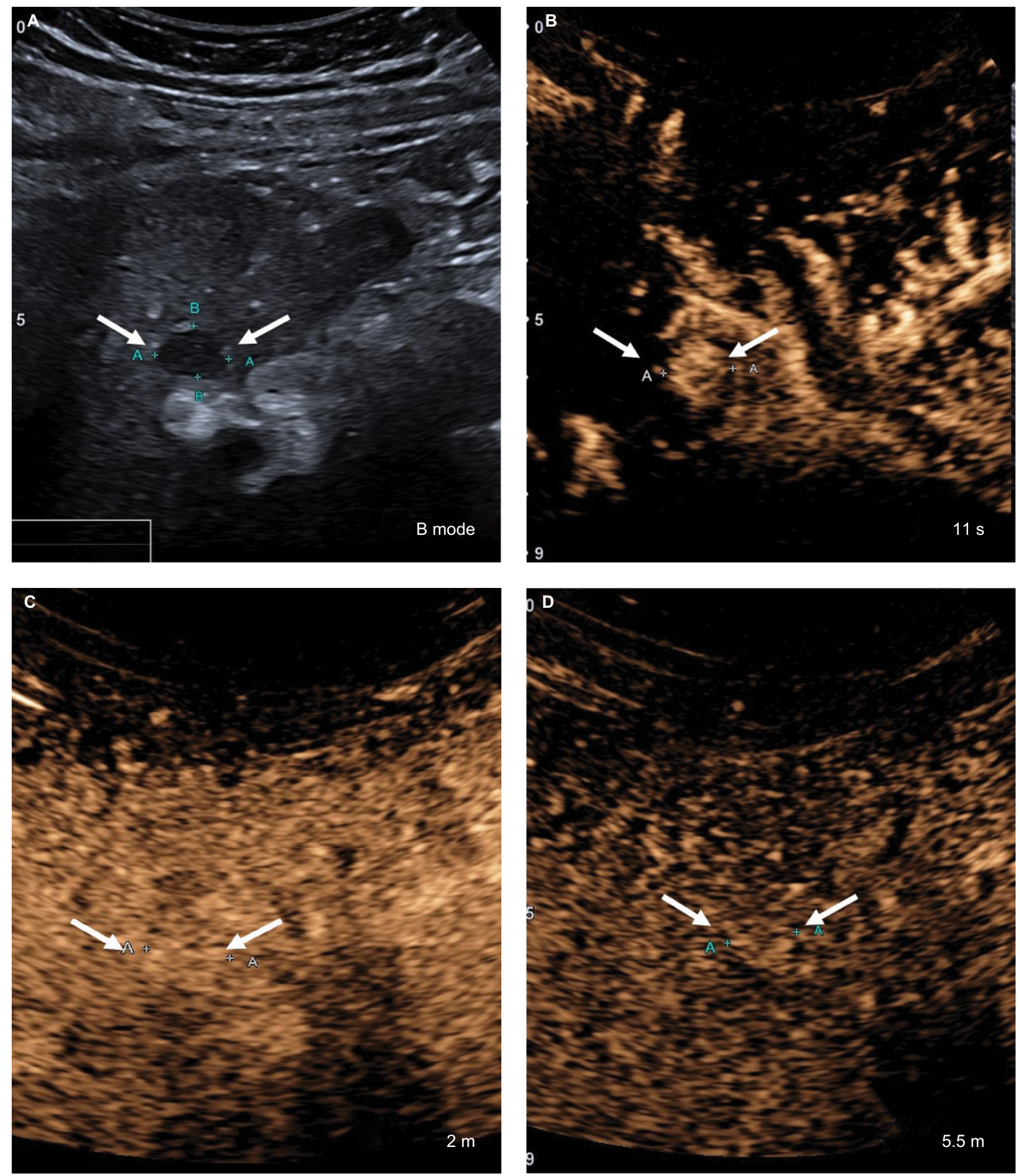

Figure 17 CEUS LR-4.

Notes: (A) A $12 \mathrm{~mm}$ hypoechoic nodule on B-mode ultrasound. (B) The entire nodule shows hyperenhancement in the arterial phase. (C) At 2 minutes, the nodule is isoenhancing compared with the surrounding liver parenchyma. (D) The nodule remains isoenhancing at 5.5 minutes without washout. Arrows show to the outline of the nodule.

Abbreviation: CEUS, contrast-enhanced ultrasound. 
show examples of different nodules with CEUS categories assigned to them.

Steps 2 (apply ancillary features) (Figure 18), 3 (apply tiebreaking rules) (Figure 19), and 4 (final check) are conceptually identical to their CT/MRI counterparts. ${ }^{39}$

\section{LI-RADS treatment response LI-RADS treatment response categories}

The LI-RADS treatment response algorithm was introduced in v2017 to guide interpretation of response following locoregional therapy and to improve clarity and consistency of communication between multidisciplinary teams managing HCC patients. $^{27}$

Response evaluation criteria in solid tumors and modified response evaluation criteria in solid tumor serve as the standard criteria for assessing treatment response in oncology clinical trials; however, these systems are not designed for use in routine practice and do not currently contribute to OPTN staging for organ allocation in liver transplantation. The LI-RADS treatment response algorithm and categories apply to observations treated by a range of locoregional therapies, but do not apply to systemic treatment response; the system can be used with caution in patients undergoing both locoregional and systemic therapy when the locoregional treatment effects are dominant. ${ }^{36}$ The treatment response algorithm defines specific imaging features/criteria for individual response categories, which are intended to communicate the probability of residual viable tumor following therapy. It is worth mentioning that these criteria primarily reflect vascularization of tissues as a surrogate for viability; this does not necessarily translate to complete pathological response due to the inability of imaging to detect microscopic

$\geq 1 \mathrm{AF}$ favoring malignancy: upgrade by 1 category up to LR-4 (absence of these AFs should not be used to downgrade)

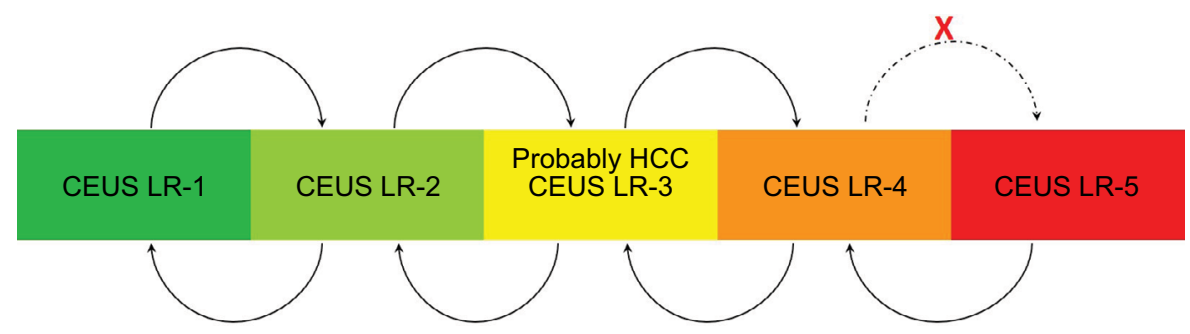

$\geq 1$ AF favoring benignity: downgrade by 1 category

Figure 18 CEUS LI-RADS AFs upgrade and downgrade of categories.

Notes: Reproduced with permission from American College of Radiology. CEUS LI-RADS v20I7. Available from: https://www.acr.org/Clinical-Resources/Reporting-andData-Systems/LI-RADS/CEUS-LI-RADS-v2017. ${ }^{39}$

Abbreviations: ACR, American College of Radiology; AF, ancillary feature; CEUS, contrast-enhanced ultrasound; LI-RADS, Liver Imaging Reporting and Data System.

If unsure about presence of TIV, do not categorize as CEUS LR-TIV

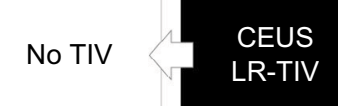

If unsure between two categories, choose the one reflecting lower certainty

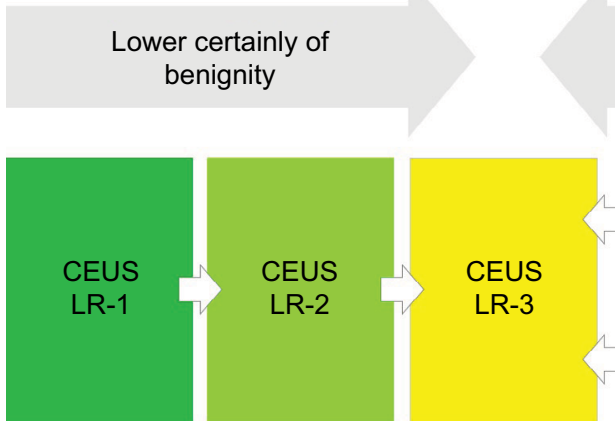

\section{Lower certainly of} malignancy

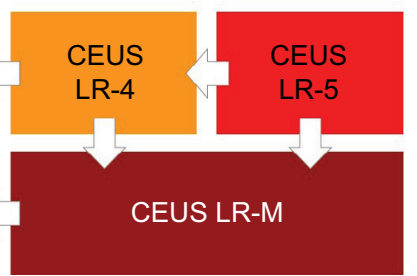

Lower certainly of hepatocellular origin

Figure 19 CEUS LI-RADS tiebreaking rules.

Notes: Reproduced with permission from American College of Radiology. CEUS LI-RADS v2017. Available from: https://www.acr.org/Clinical-Resources/Reporting-andData-Systems/LI-RADS/CEUS-LI-RADS-v2017. ${ }^{39}$

Abbreviations: ACR, American College of Radiology; CEUS, contrast-enhanced ultrasound; LI-RADS, Liver Imaging Reporting and Data System; TIV, tumor in vein. 
foci of residual tumor. ${ }^{36,42}$ The categories and their definitions are listed below and provided in Figure 20.

\section{LR-TR nonevaluable}

This category is assigned when the treatment response cannot be evaluated due to poor image quality, inadequate technique, or time interval after therapy too soon for reliable interpretation. ${ }^{36}$

\section{LR-TR nonviable}

The nonviable category should be assigned to treated lesions with no appreciable enhancement or to lesions demonstrating expected posttreatment enhancement patterns. ${ }^{16}$ For example, a common expected posttreatment enhancement pattern is thin rim enhancement that becomes progressively more intense on postarterial phases.

\section{LR-TR viable}

The viable category should be assigned to treated lesions with nodular, mass-like, or thick irregular regions of APHE, washout appearance, or enhancement similar to pretreatment. ${ }^{36}$

\section{LR-TR equivocal}

This category is applied to lesions that cannot be clearly categorized as viable or nonviable due to atypical enhancement patterns in the absence of technical or patient-related limitations. ${ }^{27}$

\section{Assigning LI-RADS treatment response categories using CT/MRI}

Similar to the diagnostic systems, the treatment response assessment is done in four steps. ${ }^{27}$

\section{Step I:Apply LI-RADS CT/MRI treatment response algorithm}

First, the reader should confirm that the imaging study is adequate, ie, without significant degradation or omission of required images. If the required images are not present, then category LR-TR nonevaluable should be assigned. ${ }^{36}$ If treatment response can be evaluated, then one of the three LR-TR categories should be assigned.

\section{Step 2: Measure observation size}

LR-TR viable and LR-TR equivocal lesions should be measured. The region of viable or potentially viable tumor is measured as the longest dimension of the enhancing tissue, without traversing the nonenhancing area. This one-dimensional measurement serves as the observation size when reporting LR-TR viable or LR-TR equivocal lesions. ${ }^{27}$ When

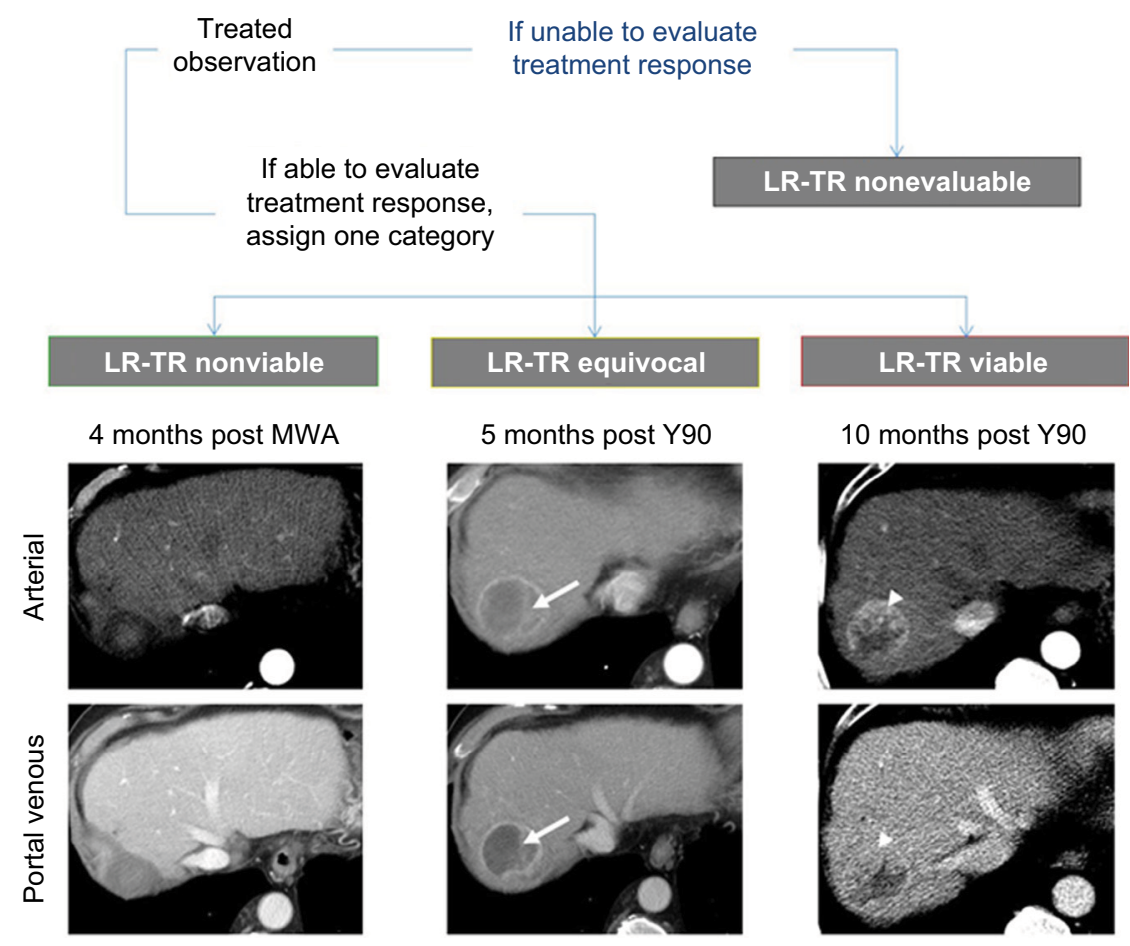

Figure 20 Treatment response categories with examples from a 70-year-old man who underwent transarterial radioembolization (Y90) followed by transarterial bland embolization and microwave ablation (MWA).

Notes: Left column shows no evidence of enhancement after MWA, categorized as LR-TR nonviable. Center column shows ill-defined enhancement (arrows) 5 months after Y90, categorized as LR-TR equivocal. Right column shows mass-like arterial phase hyperenhancement in the treated lesion (arrowheads), categorized as LR-TR viable. 
present, the size of residual viable tumor helps dictate management decisions and track tumor burden, eg, while awaiting transplantation. If there is no lesional enhancement or the enhancement pattern is expected for the applied therapy, then the appropriate treatment category is LR-TR nonviable; in this context, the size of viable tumor has no meaning and a size measurement does not need to be reported. ${ }^{15}$

\section{Steps 3 and 4:Apply tiebreaking rule and perform final check}

If the radiologist is undecided on the posttreatment category, LR-TR equivocal should be chosen to reflect the lower certainty of residual tumor. As a final check, the radiologist should question the assigned category and reevaluate if that category is inappropriate. ${ }^{15} \mathrm{CT} / \mathrm{MRI}$ diagnostic categories should still be used for untreated lesions or new lesions developing elsewhere in the liver.

\section{Conclusion}

LI-RADS has been created as a dynamic system with regular updates to maintain best practices based on latest evidence and expert multidisciplinary consensus. LI-RADS has been refined and expanded over multiple updates to now also address ultrasound-based surveillance, CEUS for HCC diagnosis, and CT/MRI for assessing treatment response after locoregional therapy in addition to $\mathrm{CT} / \mathrm{MRI}$ diagnosis. LIRADS is consistent with the National Comprehensive Cancer Network (NCCN) guidelines and can be easily converted to OPTN classes for liver transplant evaluation. The new version of LI-RADS is now fully integrated into the AASLD 2018 HCC clinical practice guidance, which represents a major step toward widespread endorsement of LI-RADS in clinical practice.

\section{Disclosure}

The authors report no conflicts of interest in this work. The views expressed in this work are those of the authors and do not necessarily reflect the official policy or position of the Department of the Navy, Department of Defense or the United States Government. Dr Robert M Marks is a military service member. This work was prepared as part of official duties. Title 17 U.S.C. 105 provides that 'Copyright protection under this title is not available for any work of the United States Government.

\section{References}

1. Marrero JA, Kulik LM, Sirlin CB, et al. Diagnosis, staging, and management of hepatocellular carcinoma: 2018 practice guidance by the American Association for the Study of Liver Diseases. Hepatology. 2018;68(2):723-750.
2. Mittal S, El-Serag HB. Epidemiology of hepatocellular carcinoma: consider the population. J Clin Gastroenterol. 2013;47 Suppl:S2-S6.

3. Forner A, Reig ME, de Lope CR, Bruix J. Current strategy for staging and treatment: the BCLC update and future prospects. Semin Liver Dis. 2010;30(1):61-74.

4. Heimbach JK, Kulik LM, Finn RS, et al. AASLD guidelines for the treatment of hepatocellular carcinoma. Hepatology. 2018;67(1):358-380.

5. Chernyak V, Santillan CS, Papadatos D, Sirlin CB. LI-RADS $®$ algorithm: CT and MRI. Abdom Radiol (NY). 2018;43(1):111-126.

6. Tang A, Valasek MA, Sirlin CB. Update on the liver imaging reporting and data system. Adv Anat Pathol. 2015;22(5):314-322.

7. American College of Radiology. Liver Imaging Reporting and Data System. Available from: https://www.acr.org/quality-safety/resources/ LIRADS. 2017. Accessed February 6, 2018.

8. Fowler KJ, Tang A, Santillan C, et al. Interreader reliability of LI-RADS version 2014 algorithm and imaging features for diagnosis of hepatocellular carcinoma: a large international multireader study. Radiology. 2018;286(1):173-185.

9. Flusberg M, Ganeles J, Ekinci T, et al. Impact of a structured report template on the quality of CT and MRI reports for hepatocellular carcinoma diagnosis. J Am Coll Radiol. 2017;14(9):1206-1211.

10. Sirlin CB, Kielar AZ, Tang A, Bashir MR. LI-RADS: a glimpse into the future. Abdom Radiol (NY). 2018;43(1):231-236.

11. American College of Radiology. Liver Imaging Reporting and Data System version; 2011. Available from: https://www.acr.org/ClinicalResources/Reporting-and-Data-Systems/LI-RADS/LI-RADS 1 . Accessed May 21, 2018.

12. American College of Radiology. Liver Imaging Reporting and Data System version; 2014 core. Available from: https://www.acr.org/ Clinical-Resources/Reporting-and-Data-Systems/LI-RADS/LI-RADSv2014. Accessed May 21, 2018.

13. Mitchell DG, Bruix J, Sherman M, Sirlin CB. LI-RADS (Liver Imaging Reporting and Data System): summary, discussion, and consensus of the LI-RADS Management Working Group and future directions. Hepatology. 2015;61(3):1056-1065.

14. Wald C, Russo MW, Heimbach JK, Hussain HK, Pomfret EA, Bruix J. New OPTN/UNOS policy for liver transplant allocation: standardization of liver imaging, diagnosis, classification, and reporting of hepatocellular carcinoma. Radiology. 2013;266(2):376-382.

15. American College of Radiology. Liver Imaging Reporting and Data System version 2017 core. Available from: https://www.acr.org/ClinicalResources/Reporting-and-Data-Systems/LI-RADS. Accessed May 20, 2018.

16. American College of Radiology. Liver Imaging Reporting and Data System version 2018 core. Available from: https://www.acr.org/ClinicalResources/Reporting-and-Data-Systems/LI-RADS. Accessed May 21, 2018.

17. Tzartzeva K, Obi J, Rich NE, et al. Surveillance imaging and alpha detoprotein for early detection of hepatocellular carcinoma in patients with cirrhosis: a meta-analysis. Gastroenterology. 2018;154(6):1706-1718.e1.

18. Zhang BH, Yang BH, Tang ZY. Randomized controlled trial of screening for hepatocellular carcinoma. J Cancer Res Clin Oncol. 2004;130(7):417-422.

19. Yeh YP, Hu TH, Cho PY, et al; Changhua Community-Based Abdominal Ultrasonography Screening Group. Evaluation of abdominal ultrasonography mass screening for hepatocellular carcinoma in Taiwan. Hepatology. 2014;59(5):1840-1849.

20. Morgan TA, Maturen KE, Dahiya N, Sun MRM, Kamaya A; American College of Radiology Ultrasound Liver Imaging and Reporting Data System (US LI-RADS) Working Group. US LI-RADS: ultrasound liver imaging reporting and data system for screening and surveillance of hepatocellular carcinoma. Abdom Radiol (NY). 2018;43(1):41-55.

21. American College of Radiology. Ultrasound LI-RADS v2017. Available from: https://www.acr.org/Clinical-Resources/Reporting-and-DataSystems/LI-RADS/Ultrasound-LI-RADS-v2017. Accessed May 21, 2018.

22. Tang A, Hallouch O, Chernyak V, Kamaya A, Sirlin CB. Epidemiology of hepatocellular carcinoma: target population for surveillance and diagnosis. Abdom Radiol (NY). 2018;43(1):13-25. 
23. Vilgrain V, Lewin M, Vons $\mathrm{C}$, et al. Hepatic nodules in Budd-Chiari syndrome: imaging features. Radiology (NY). 1999;210(2):443-450.

24. Flor N, Zuin M, Brovelli F, et al. Regenerative nodules in patients with chronic Budd-Chiari syndrome: a longitudinal study using multiphase contrast-enhanced multidetector CT. Eur J Radiol. 2010;73(3):588-593.

25. Santillan CS, Tang A, Cruite I, Shah A, Sirlin CB. Understanding LIRADS: a primer for practical use. Magn Reson Imaging Clin $\mathrm{N} \mathrm{Am}$. 2014;22(3):337-352.

26. Efremidis SC, Hytiroglou P. The multistep process of hepatocarcinogenesis in cirrhosis with imaging correlation. Eur Radiol. 2002;12(4):753-764.

27. Elsayes KM, Hooker JC, Agrons MM, et al. 2017 Version of LI-RADS for CT and MR imaging: an update. Radiographics. 2017;37(7): 1994-2017.

28. Marrero JA, Hussain HK, Nghiem HV, Umar R, Fontana RJ, Lok AS. Improving the prediction of hepatocellular carcinoma in cirrhotic patients with an arterially-enhancing liver mass. Liver Transpl. 2005;11(3): 281-289.

29. Choi JY, Lee JM, Sirlin CB. CT and MR imaging diagnosis and staging of hepatocellular carcinoma: part II. Extracellular agents, hepatobiliary agents, and ancillary imaging features. Radiology. 2014;273(1):30-50.

30. Liu YI, Shin LK, Jeffrey RB, Kamaya A. Quantitatively defining washout in hepatocellular carcinoma. AJR Am J Roentgenol. 2013;200(1):84-89.

31. Ishigami K, Yoshimitsu K, Nishihara Y, et al. Hepatocellular carcinoma with a pseudocapsule on gadolinium-enhanced MR images: correlation with histopathologic findings. Radiology. 2009;250(2):435-443.

32. Kadoya M, Matsui O, Takashima T, Nonomura A. Hepatocellular carcinoma: correlation of MR imaging and histopathologic findings. Radiology. 1992;183(3):819-825.
33. Seuss CR, Kim MJ, Triolo MJ, Hajdu CH, Rosenkrantz AB. Comparison of MRI pulse sequences for prediction of size of hepatocellular carcinoma at explant evaluation. AJR Am J Roentgenol. 2014;203(2):300-305.

34. Chernyak V, Tang A, Flusberg M, et al. LI-RADS $®$ ancillary features on CT and MRI. Abdom Radiol (NY). 2018;43(1):82-100.

35. Fowler KJ, Potretzke TA, Hope TA, Costa EA, Wilson SR, Li-Rads M. LI-RADS M (LR-M): definite or probable malignancy, not specific for hepatocellular carcinoma. Abdom Radiol (NY). 2018;43(1):149-157.

36. Kielar A, Fowler KJ, Lewis S, et al. Locoregional therapies for hepatocellular carcinoma and the new LI-RADS treatment response algorithm. Abdom Radiol (NY). 2018;43(1):218-230.

37. Colagrande S, Centi N, Galdiero R, Ragozzino A. Transient hepatic intensity differences: part 1 , those associated with focal lesions. AJR Am J Roentgenol. 2007;188(1):154-159.

38. Santillan C, Chernyak V, Sirlin C. LI-RADS categories: concepts, definitions, and criteria. Abdom Radiol (NY). 2018;43(1):101-110.

39. American College of Radiology. CEUS LI-RADS v2017. Available from: https:/www.acr.org/Clinical-Resources/Reporting-and-Data-Systems/ LI-RADS/CEUS-LI-RADS-v2017. Accessed May 22, 2018.

40. Wilson SR, Lyshchik A, Piscaglia F, et al. CEUS LI-RADS: algorithm, implementation, and key differences from CT/MRI. Abdom Radiol (NY). 2018;43(1):127-142.

41. Kono Y, Lyshchik A, Cosgrove D, et al. Contrast Enhanced Ultrasound (CEUS) Liver Imaging Reporting and Data System (LI-RADS $®)$ : the official version by the American College of Radiology (ACR). Ultraschall Med. 2017;38(1):85-86.

42. Becker-Weidman D, Civan JM, Deshmukh SP, et al. Hepatocellular carcinoma after locoregional therapy: magnetic resonance imaging findings in falsely negative exams. World J Hepatol. 2016;8(16):685-690.
Journal of Hepatocellular Carcinoma

\section{Publish your work in this journal}

The Journal of Hepatocellular Carcinoma is an international, peerreviewed, open access journal that offers a platform for the dissemination and study of clinical, translational and basic research findings in this rapidly developing field. Development in areas including, but not limited to, epidemiology, vaccination, hepatitis therapy, pathology and

\section{Dovepress}

molecular tumor classification and prognostication are all considered for publication. The manuscript management system is completely online and includes a very quick and fair peer-review system, which is all easy to use. Visit http://www.dovepress.com/testimonials.php to read real quotes from published authors. 